January 9, 2008

\title{
Global-scale seismic interferometry: theory and numerical examples
}

\author{
Elmer Ruigrok, Deyan Draganov and Kees Wapenaar \\ Department of Geotechnology \\ Delft University of Technology \\ P.O. Box 5048 \\ 2600 GA Delft \\ The Netherlands \\ E-mail: e.n.ruigrok@tudelft.nl
}

Geophysical Prospecting paper submitted for the special issue on 'What Can EP learn from Seismology and Vice-versa?' 


\begin{abstract}
Progress in the imaging of the mantle and core is partially limited by the sparse distribution of natural sources; the earthquake hypocenters are mainly along the active lithospheric plate boundaries. This problem can be approached with seismic interferometry (SI). In recent years, there has been considerable progress in the development of seismic interferometric techniques. The term seismic interferometry refers to the principle of generating new seismic responses by cross-correlating seismic observations at different receiver locations. The application of interferometric techniques on a global scale could create sources at locations where no earthquakes occur. In this way, yet unknown responses would become available for the application of travel-time tomography and surface-wave dispersion studies. The retrieval of a dense-enough sampling of source gathers would largely benefit the application of reflection imaging.
\end{abstract}

We derive new elastodynamic representation integrals for global-scale seismic interferometry. The relations are different from other SI relations for transient sources, in the sense that they are suited for a rotating closed system like the Earth. We use a correlation of an observed response with a response to which free-surface multiple elimination has been applied to account for the closed system. Despite the fact that the rotation of the Earth breaks source-receiver reciprocity, the SI relations are shown to be valid. The Coriolis force is included without the need to evaluate an extra term.

We synthesize global-scale earthquake responses and use them to illustrate the acoustic versions of the new interferometric relations. When the sampling of real source locations is dense enough, then both the response with and the response without free-surface multiples are retrieved. When we do not take into account the responses from sources in the direct neighborhood of the SI-constructed source location, still the response with free-surface multiples can be retrieved. Even when only responses from sources at a certain range of epicentral distances are available, some events in the Green's function between two receiver locations can still be retrieved. The retrieved responses are not perfect, but the artifacts can largely be ascribed to numerical errors. The reconstruction of internal events - the response as if there is a source and a receiver on (major) contrasts within the model - could possibly be of use for imaging.

With modeling it is possible to find out in what region of the correlation panel stationary 
phases occur that contribute to the retrieval of events. This knowledge opens up a new way of filtering out undesired events and of finding out whether specific events could be retrieved with a given source-receiver configuration. 


\section{KEYWORDS}

global-scale seismology, wave propagation, cross-correlation, Green's function retrieval, body waves 


\section{INTRODUCTION}

Seismic reflection imaging (SRI) has proven to be the most successful technique for exploration of oil and gas. For the application of this technique, the subsurface needs to be illuminated from many different angles. With the acquired responses a high-resolution image of the subsurface can be made. On the exploration scale, the illumination from many directions can be achieved by using a dense grid of controlled sources at the surface (dynamite, vibrators, air guns). On a global scale, though, one is dependent on the natural-source distribution. Only the larger earthquakes generate enough energy to illuminate the deep interior of the Earth. These larger earthquakes are mainly situated along the active lithospheric plate boundaries (Fig. 1). Thus, there are many areas on the globe, beneath which the Earth's interior is not directly illuminated. It is especially this sparse source distribution that impedes the use of SRI on a global scale. Also, to get a better laterally varying velocity model using travel-time tomography or surface-wave dispersion studies, more source locations are required. The shortage of source locations on a global scale can be reduced with Seismic Interferometry (SI).

In recent years, there has been considerable progress in the development of SI techniques, see the July/August 2006 issue of Geophysics for an overview. SI is a method by which additional information can be extracted from wavefield responses. The responses measured at any combination of two receiver locations can be combined with SI to construct the response as if there were a source at one of the receiver locations and a receiver at the other. In this way, theoretically, a source can be created at any receiver position, provided there is enough illumination.

SI relations have been derived for two exploration-type configurations. The first configuration, for transient or noise sources in the subsurface, was introduced by Claerbout (1968) for 1D media and was proven to hold for a 3D arbitrary inhomogeneous medium by Wapenaar et al. (2002). These relations (for transient and for noise sources) were numerically validated by Draganov et al. (2004b) and successfully applied on real data by Draganov et al. (2007) for noise sources and by Shiraishi et al. (2006) for transient sources.

The other configuration, for controlled sources at the surface, was introduced by Schuster (2001). The technique, including an imaging step, was successfully applied on real data (Schuster et al., 2004). For the situation with receivers in a borehole, Bakulin \& Calvert 
(2004) developed the 'virtual source method', by which the sources at the surface are redatumed through a complex overburden to the receiver positions in the borehole.

Independent of the developments in exploration geophysics, SI was also introduced in the solid Earth community. The work was inspired by ultrasonic experiments performed by Weaver \& Lobkis (2001) and by time-reversed acoustics experiments (Derode et al., 2003). Campillo \& Paul (2003) used SI to construct surface-wave Green's functions between pairs of station positions. They used the diffusive part of the coda information from earthquake responses. Other researchers (Gerstoft et al., 2006; Shapiro et al., 2005; Yao et al., 2006) also applied SI to retrieve surface-wave Green's functions on a regional scale. They used different source types and correlation procedures. The retrieved Green's functions can directly be used to estimate velocity models of the crust and upper mantle with travel-time tomography and surface-wave dispersion studies.

Another application of SI in solid Earth research was introduced by Schuster et al. (2004). They showed a synthetic example of applying SI and an imaging step on scattered teleseismic arrivals. They used the first reverberations of an incoming wavefront between the free surface and large crustal interfaces. This application has the same configuration as the explorationscale case with transient or noise sources in the subsurface. The main difference is the scale. Also Shragge et al. (2006), Fan et al. (2006), Kumar \& Bostock (2006) and Abe et al. (2007) applied SI on regional-scale seismology using scattered teleseismic arrivals.

SI has not yet been applied in global-scale body-wave seismology. The application of SI on a global scale would reconstruct the responses from sources at locations where no earthquakes occur. Since the locations of the receivers are exactly known, so are the locations of the retrieved sources. This is not the case for real earthquakes, for which there is an uncertainty of the exact source locations. For example, SI could be applied on data from the USArray, of which the design is discussed in Levander (2003). Using SI, new source positions could be retrieved at any of the receiver positions. In this way, yet unknown responses become available for the application of travel-time tomography. The retrieval of a dense-enough sampling of source gathers would largely benefit the application of SRI, with which a much better image of the deep interior could be obtained.

As noted before, exact SI relations for transient sources were derived for exploration-type configurations (Wapenaar \& Fokkema, 2006). Wave phenomena on the scale of the entire Earth cannot be described by these configurations. The main reason is the presence of a 
closed free surface, which traps the energy.

Weaver \& Lobkis (2001) derived an interferometric relation for a diffuse wavefield in a closed system. They performed an experiment with random sources (acoustic thermal fluctuations in an ultrasonic experiment) placed throughout a volume. The Earth is not filled with random sources, at least not with sources exciting waves in the frequency bandwidth that is used in global seismology. Hence, the wavefield in the Earth is far from diffuse. Moreover, the noise sources near the surface (ocean-wave motion, etc.) do not excite enough energy to illuminate the very deep contrasts like the Core-Mantle Boundary (CMB).

In this paper, we derive elastodynamic global-scale interferometric relations for transient sources (earthquakes) near the enclosing surface of the medium (the Earth's free surface). In this derivation, we take the rotation of the Earth into account. The acoustic versions of the relations are numerically validated with synthesized global-scale earthquake responses. Besides illustrating the derived relations numerically, we also use the synthesized responses to test one of the relations for more realistic situations, namely suboptimal source sampling and responses only from sources at a specific range of epicentral distances.

The terminology used in exploration-scale seismology is sometimes different from that used in solid Earth seismology (Table I). In this paper, no explicit choice has been made for one of the two. But to avoid confusion we consequently use the exploration terms 'source' and 'event' instead of solid-Earth terms 'seismic event' and 'phase', respectively.

\section{DERIVATION OF GLOBAL-SCALE SI RELATIONS}

In this section, we derive and clarify global-scale SI relations for transient sources near the surface. First the correlation-type reciprocity theorem is derived. Subsequently it is used to derive a global-scale SI relation that takes the rotation of the Earth and the Coriolis force into account. Afterwards we make a small adjustment to handle the absence of near-offset responses, as is common in the real Earth situation. With near-offset responses we mean the responses due to the sources which are in the direct neighborhood of the receiver location where we want to reconstruct a source. In the end of this section we present the acoustic versions of the derived relations. 


\subsection{Elastodynamic reciprocity theorem}

We start with the derivation of the correlation-type elastodynamic reciprocity theorem in the frequency domain. A similar derivation (but without Coriolis force) can be found in Wapenaar \& Fokkema (2006). The temporal Fourier transform of a space- and timedependent quantity $p(\mathbf{x}, t)$ is defined as

$$
\hat{p}(\mathbf{x}, \omega)=\int_{-\infty}^{\infty} p(\mathbf{x}, t) e^{-j \omega t} d t
$$

where $j$ is the imaginary unit and $\omega$ is the angular frequency. The hat above the quantities denotes that they are in the frequency domain. $\mathbf{x}=\left(x_{1}, x_{2}, x_{3}\right)$ denotes the position vector.

A reciprocity theorem relates two independent states in one and the same domain (de Hoop, 1988; Fokkema \& van den Berg, 1993). Here we relate two elastodynamic states denoted with $A$ and $B$. A state is characterized by the source state, the material state and the wavefield state. The source state defines the types, locations and timings of sources within the domain $\mathbb{D}$. The material state gives the relevant material properties within $\mathbb{D}$. In the elastodynamic case, these properties are mass density $\rho$ and compliance $s_{i j k l}$. The wavefield state is an expression of the waves that exist within $\mathbb{D}$ due to sources within, on the boundary of, or outside the domain $\mathbb{D}$. The wavefield is characterized by the stress tensor $\tau_{i j}(\mathbf{x}, t)$ and the particle velocity $v_{i}(\mathbf{x}, t)$. The stress tensor and the particle velocity are coupled by the equation of motion, written in the space-frequency domain as (Dahlen \& Tromp, 1998)

$$
j \omega \hat{\rho} \hat{v}_{i}-\partial_{j} \hat{\tau}_{i j}+2 \hat{\rho} \epsilon_{i j k} \Omega_{j} \hat{v}_{k}=\hat{f}_{i},
$$

and the linearized stress-strain relation

$$
-j \omega \hat{s}_{i j k l} \hat{\tau}_{k l}+\left(\partial_{j} \hat{v}_{i}+\partial_{i} \hat{v}_{j}\right) / 2=\hat{h}_{i j}
$$

where $\partial_{j}$ denotes differentiation with respect to the spatial coordinates. $\epsilon_{i j k}$ is the LeviCevita operator, $\epsilon_{i j k}=1$ when $i j k=123 ; 231 ; 312, \epsilon_{i j k}=-1$ when $i j k=132 ; 213 ; 321$, while $\epsilon_{i j k}=0$ otherwise. For repeated Latin subscripts Einstein's summation convention applies. In (2), the third term on the left-hand side is the apparent Coriolis force for a solid rotation with angular velocity $\Omega_{j}$ and $\hat{f}_{i}(\mathbf{x}, \omega)$ is the external volume force density. In (3) $\hat{h}_{i j}(\mathbf{x}, \omega)$ is the external deformation rate density. In (2) and (3) the medium parameters $\hat{\rho}$ and $\hat{s}_{i j k l}$ are for the moment frequency dependent and complex valued and thus account for inelastic losses in the medium. 
We use the integral theorem of Gauss for any continuously differentiable vector function $\hat{p}_{j}:$

$$
\oint_{\partial \mathbb{D}} \hat{p}_{j} n_{j} d^{2} \mathbf{x}=\int_{\mathbb{D}} \partial_{j} \hat{p}_{j} d^{3} \mathbf{x}
$$

where $\partial \mathbb{D}$ is the boundary of $\mathbb{D}$ and $n_{j}$ is the outward-pointing unit vector normal to $\partial \mathbb{D}$ (Fig. $2), d^{3} \mathbf{x}$ the elementary volume in 3 -dimensional Euclidean space and $d^{2} \mathbf{x}$ the elementary area of $\partial \mathbb{D}$.

For $\hat{p}_{j}$ we choose an interaction function between the wavefield quantities of two elastodynamic states, denoted with $A$ and $B$, respectively:

$$
\hat{p}_{j}=-\hat{v}_{i, A}^{*} \hat{\tau}_{i j, B}-\hat{\tau}_{i j, A}^{*} \hat{v}_{i, B}
$$

in which * denotes complex conjugation and $\hat{\tau}_{i j, A} / \hat{\tau}_{i j, B}$ and $\hat{v}_{i, A} / \hat{v}_{i, B}$ denote the stress tensor and particle velocity of state $A / B$, respectively. Substituting equation (5) into Gauss' integral theorem, applying the product rule for differentiation for the integrand of the volume integral, substituting (2) and (3) or their complex conjugated versions to replace $\partial_{j} \hat{\tau}_{i j, A}^{*}$, $\partial_{j} \hat{\tau}_{i j, B}, \partial_{j} \hat{v}_{i, A}^{*}$ and $\partial_{j} \hat{v}_{i, B}$, using the symmetry relations $s_{i j k l}=s_{k l i j}$ and $\tau_{i j}=\tau_{j i}$ and finally reordering the terms in integrals containing medium-parameter terms, source terms and Coriolis terms, yields

$$
\begin{aligned}
& \oint_{\partial \mathbb{D}}\left\{-\hat{v}_{i, A}^{*} \hat{\tau}_{i j, B}-\right.\left.\hat{\tau}_{i j, A}^{*} \hat{v}_{i, B}\right\} n_{j} d^{2} \mathbf{x}= \\
&-j \omega \int_{\mathbb{D}}\left\{\hat{\tau}_{i j, A}^{*}\left(\hat{s}_{i j k l, B}-\hat{s}_{i j k l, A}^{*}\right) \tau_{k l, B}+\hat{v}_{i, A}^{*}\left(\hat{\rho}_{B}-\hat{\rho}_{A}^{*}\right) \hat{v}_{i, B}\right\} d^{3} \mathbf{x} \\
& \quad+\int_{\mathbb{D}}\left\{-\hat{\tau}_{i j, A}^{*} \hat{h}_{i j, B}+\hat{v}_{i, A}^{*} \hat{f}_{i, B}-\hat{h}_{i j, A}^{*} \hat{\tau}_{i j, B}+\hat{f}_{i, A}^{*} \hat{v}_{i, B}\right\} d^{3} \mathbf{x} \\
& \quad-\int_{\mathbb{D}}\left\{2 \hat{\rho}_{A}^{*} \epsilon_{i j k} \hat{v}_{i, B} \Omega_{j, A} \hat{v}_{k, A}^{*}+2 \hat{\rho}_{B} \epsilon_{i j k} \hat{v}_{i, A}^{*} \Omega_{j, B} \hat{v}_{k, B}\right\} d^{3} \mathbf{x} .
\end{aligned}
$$

Equation (6) is the Rayleigh-Betti reciprocity theorem of the correlation type accounting for Coriolis force.

From here onward we take the material states for state $A$ and $B$ equal and we consider lossless media. Hence $\hat{\rho}_{A}^{*}=\hat{\rho}_{B}=\rho$ and $\hat{s}_{i j k l, A}^{*}=\hat{s}_{i j k l, B}=s_{i j k l}$. As a result the integral with the medium-contrast interactions in (6) - the first integral on the right-hand side of the equation - vanishes.

For the application to global-scale wave phenomena we choose $\Omega_{j, A}=\Omega_{j, B}=\Omega_{j}$, which is the solid rotation of the Earth. The integral with the Coriolis terms in (6) - the third 
integral on the right-hand side of the equation - can now be rewritten as

$$
-\int_{\mathbb{D}}\left\{2 \rho\left(\epsilon_{i j k} \hat{v}_{i, B} \Omega_{j} \hat{v}_{k, A}^{*}+\epsilon_{i j k} \hat{v}_{i, A}^{*} \Omega_{j} \hat{v}_{k, B}\right)\right\} d^{3} \mathbf{x} .
$$

Using the property $\epsilon_{i j k}=-\epsilon_{k j i}$ we may rewrite the last term under the integral as $-\epsilon_{i j k} \hat{v}_{k, A}^{*} \Omega_{j} \hat{v}_{i, B}$. Hence the third integral on the right-hand side of (6) vanishes as well.

Consequently, for a solid rotating lossless Earth the Rayleigh-Betti reciprocity theorem of the correlation type accounting for Coriolis force becomes

$$
\begin{aligned}
\oint_{\partial \mathbb{D}}\left\{-\hat{v}_{i, A}^{*} \hat{\tau}_{i j, B}-\hat{\tau}_{i j, A}^{*} \hat{v}_{i, B}\right\} n_{j} d^{2} \mathbf{x}= & \\
& \int_{\mathbb{D}}\left\{-\hat{\tau}_{i j, A}^{*} \hat{h}_{i j, B}+\hat{v}_{i, A}^{*} \hat{f}_{i, B}-\hat{h}_{i j, A}^{*} \hat{\tau}_{i j, B}+\hat{f}_{i, A}^{*} \hat{v}_{i, B}\right\} d^{3} \mathbf{x},
\end{aligned}
$$

in which - surprisingly - no term is needed to account for the Coriolis force.

Fokkema \& van den Berg (1993) related different acoustic states with the help of the acoustic version of (8), without considering rotation. Doing so, useful relations were derived for seismic applications. Following a similar procedure, both acoustic and elastodynamic relations for SI were derived by Wapenaar \& Fokkema (2006). Snieder (2007) used the acoustic version of (6), without considering rotation and without a complex density, but with a complex-valued compressibility to derive interferometric relations for dissipative acoustic media.

\subsection{Basic relation}

The representations for state $A$ and state $B$ are depicted in Fig. 3. States $A$ and $B$ are so-called Green's states: the sources are assumed to be impulses, such that the responses can be written as impulse responses or Green's functions. To link reality with a Green's state, a wavefield registration would need to be deconvolved for the source wavelet.

Fig. 3(a) depicts the representation for State $A$. Domain $\mathbb{D}$ covers the entire inner space of the Earth. The boundary surface $\partial \mathbb{D}$ of $\mathbb{D}$ coincides with the Earth's surface. A shallowdepth earthquake (source) has a hypocenter at $\mathbf{x}_{A}$, which is approximately at the Earth's surface. The source in state $A$ is represented as a boundary condition for the traction at the free surface (which is abbreviated as FS throughout the remainder of this text). The boundary condition for approaching the FS from its interior (Earth) is

$$
\lim _{\mathbf{x} \uparrow \mathbf{x}_{\mathbf{F}} \mathbf{S}} \hat{\tau}_{i k}(\mathbf{x}) n_{k}=\delta\left(\mathbf{x}-\mathbf{x}_{A}\right) \delta_{i p} \text { for } \mathbf{x} \in \partial \mathbb{D}
$$


in which $n_{k}$ denotes the outward pointing normal vector as depicted in Fig 2 and $\delta_{i p}$ is the Kronecker delta function, $\delta_{i p}=0$ for $i \neq p, \delta_{i p}=1$ for $i=p$. The Dirac delta function on the right-hand side of (9) is a $2 \mathrm{D}$ delta function in the tangential directions.

Fig. 3(b) depicts the representation for State B. A shallow-depth earthquake has a hypocenter at $\mathbf{x}_{B}$, which is just within $\mathbb{D}$. This earthquake is assumed to be a deformationrate source. State $B$ is a representation for an Earth without FS. The response of state $B$ can be obtained from a real Earth response by using, e.g., surface-related multiple elimination (SRME) (Berkhout \& Verschuur, 1997; Verschuur \& Berkhout, 1997).

The choices made above for the type of earthquake sources is not unique. Other source mechanisms can be dealt with, but will not be considered in this paper.

The choice to include one state with FS and one without FS is not arbitrary. If we chose both states to include a FS there would be no integral left to be evaluated in (8). If we removed the FS in both states, then there would be no reflections at the FS, which are necessary for the retrieval of a response with SI.

States $A$ and state $B$ are representations of an Earth that is rotating in the opposite direction as compared to the actual rotation of the Earth $\Omega_{j}$ (Fig. 3). This choice will become clear later.

The mathematical expressions for the two elastodynamic states are listed in Table II. States $A$ and $B$ are defined for one and the same lossless inhomogeneous elastodynamic medium. The wavefield quantities, $\hat{\tau}_{i j}$ and $\hat{v}_{i}$, can be represented as Green's functions everywhere within $\mathbb{D}$ and for state $B$ also everywhere at $\partial \mathbb{D}$. In state $A$, at the $\mathrm{FS}$, we can only have a traction vector $\hat{\tau}_{i k} n_{k}$ unequal to zero at the location of the source. However, because the FS can move freely, $\hat{v}_{i}$ can be non-zero everywhere at $\partial \mathbb{D}$. The source in state $B$ is represented in the source state. Here the Dirac delta function is a $3 \mathrm{D}$ delta function.

The first and second superscript of the Green's function $\hat{G}$ denote, respectively, the observed response type $(\tau$ - stress tensor; $v$ - particle velocity) and the source type $(\tau$ traction source; $h$ - deformation-rate source). The subscripts denote the components of the observed response and the source, respectively. When only one subscript belongs to the traction or deformation-rate source, it is a traction or deformation-rate vector acting on a plane tangential to the FS. The bar in state $B$ denotes a response without FS multiples. When the bar is omitted (as in state $A$ ) we mean the complete Green's function, including all FS multiples. $\hat{G}\left(\mathbf{x}, \mathbf{x}_{A}, \omega \mid-\boldsymbol{\Omega}\right)$ is the response observed at $\mathbf{x}$ due to a source at $\mathbf{x}_{A}$, 
given as a function of frequency $\omega$, for a medium rotating with minus the angular velocity of the Earth, $-\Omega$.

We substitute the expressions of Table II into (8). Making use of the sifting property of the delta function and the multiplication property of the Kronecker delta function, we obtain

$$
\begin{aligned}
-\oint_{\partial \mathbb{D}}\left\{\hat{G}_{i, p}^{v, \tau}\left(\mathbf{x}, \mathbf{x}_{A}, \omega \mid-\Omega\right)\right\}^{*} & \hat{\bar{G}}_{i, q r}^{\tau, h}\left(\mathbf{x}, \mathbf{x}_{B}, \omega \mid-\Omega\right) d^{2} \mathbf{x}= \\
& -\left\{\hat{G}_{q,, p}^{\tau, \tau}\left(\mathbf{x}_{B}, \mathbf{x}_{A}, \omega \mid-\Omega\right)\right\}^{*}+\hat{\bar{G}}_{p, q r}^{v, h}\left(\mathbf{x}_{A}, \mathbf{x}_{B}, \omega \mid-\boldsymbol{\Omega}\right) .
\end{aligned}
$$

We change the integration over receiver positions to an integration over source positions by applying the generalized source-receiver reciprocity relations for the Green's functions. In a rotating medium the usual source-receiver reciprocity relation breaks down, hence

$$
\hat{G}\left(\mathbf{x}_{B}, \mathbf{x}_{A}, \omega \mid \mathbf{\Omega}\right) \neq \hat{G}\left(\mathbf{x}_{A}, \mathbf{x}_{B}, \omega \mid \mathbf{\Omega}\right)
$$

However, in appendix A it is shown that generalized source-receiver reciprocity relations, with opposite rotation, do hold:

$$
\hat{G}\left(\mathbf{x}_{B}, \mathbf{x}_{A}, \omega \mid \Omega\right)=\hat{G}\left(\mathbf{x}_{A}, \mathbf{x}_{B}, \omega \mid-\Omega\right) .
$$

Thus using the generalized source-receiver reciprocity relations (30), (33), (28) and (32) and subsequently applying the inverse Fourier transform, we obtain the following expression in the time domain:

$$
\begin{aligned}
\oint_{\partial \mathbb{D}} G_{p, i}^{v, \tau}\left(\mathbf{x}_{A}, \mathbf{x},-t \mid \boldsymbol{\Omega}\right) * \bar{G}_{q r, i}^{\tau, h}\left(\mathbf{x}_{B}, \mathbf{x}, t \mid \boldsymbol{\Omega}\right) d^{2} \mathbf{x}= \\
\\
G_{p, q r}^{v, h}\left(\mathbf{x}_{A}, \mathbf{x}_{B},-t \mid \boldsymbol{\Omega}\right)-\bar{G}_{q, p, p}^{\tau, f}\left(\mathbf{x}_{B}, \mathbf{x}_{A}, t \mid \mathbf{\Omega}\right),
\end{aligned}
$$

in which $*$ denotes convolution. Equation (13) is the basic global-scale SI relation for the actual rotating Earth. It states that we can reconstruct Green's functions between receiver position $\mathbf{x}_{A}$ and $\mathbf{x}_{B}$ in a rotating Earth when we cross-correlate responses at $\mathbf{x}_{A}$ and $\mathbf{x}_{B}$ resulting from sources at all $\mathbf{x}$ on $\partial \mathbb{D}$ and subsequently integrate along these sources.

We have shown here that the Green's function retrieval is in principle also valid for rotating media. Similar to flow, as discussed by Wapenaar (2006), rotation breaks time reversal and source-receiver reciprocity, but does not break Green's function retrieval by SI. 


\subsection{Relation without near-offset responses}

In the previous section, we derived an SI relation that contains an integration over responses from sources all around the globe. In reality, one would like to simulate a source on a place where no earthquakes occur. This would mean that at least the near-offset responses are missing.

To evaluate what happens if we cannot integrate over the near-offset part, we divide the integration surface $\partial \mathbb{D}$ in a near-offset part $\partial \mathbb{D}_{0}$ with respect to $\mathbf{x}_{A}$ and the remaining surface $\partial \mathbb{D}_{1}$ :

$$
\partial \mathbb{D}=\partial \mathbb{D}_{0}+\partial \mathbb{D}_{1}
$$

We substitute the expressions of Table II into (8), apply the sifting property for the Delta function and the multiplication property of the Kronecker Delta function in the volume integral and replace the complete surface integral by an integration over $\partial \mathbb{D}_{1}$, yielding

$$
\begin{aligned}
& \int_{\partial \mathbb{D}_{1}} \delta\left(\mathbf{x}-\mathbf{x}_{A}\right) \delta_{i p} \hat{\bar{G}}_{i, q r}^{v, h}\left(\mathbf{x}, \mathbf{x}_{B}, \omega \mid-\mathbf{\Omega}\right) d^{2} \mathbf{x} \\
&+\int_{\partial \mathbb{D}_{1}}\left\{\hat{G}_{i, p}^{v, \tau}\left(\mathbf{x}, \mathbf{x}_{A}, \omega \mid-\mathbf{\Omega}\right)\right\}^{*} \hat{\bar{G}}_{i, q r}^{\tau, h}\left(\mathbf{x}, \mathbf{x}_{B}, \omega \mid-\mathbf{\Omega}\right) d^{2} \mathbf{x} \approx \\
&\left\{\hat{G}_{q r, p}^{\tau, \tau}\left(\mathbf{x}_{B}, \mathbf{x}_{A}, \omega \mid-\mathbf{\Omega}\right)\right\}^{*} .
\end{aligned}
$$

By not including the integration over $\partial \mathbb{D}_{0}$, we do not perfectly satisfy the representation in Table II. Thus (15) is not exact.

Since $\partial \mathbb{D}_{1}$ does not include $\mathbf{x}_{A}, \delta\left(\mathbf{x}-\mathbf{x}_{A}\right)$ is zero for all $\mathbf{x} \epsilon \partial \mathbb{D}_{1}$ and the first term on the left-hand side of (15) vanishes. We apply generalized source-receiver reciprocity relations for Green's functions (30), (33) and (28). Subsequently applying the inverse Fourier transform, we obtain the following expression in the time domain:

$$
\int_{\partial \mathbb{D}_{1}} G_{p, i}^{v, \tau}\left(\mathbf{x}_{A}, \mathbf{x},-t \mid \mathbf{\Omega}\right) * \bar{G}_{q r, i}^{\tau, h}\left(\mathbf{x}_{B}, \mathbf{x}, t \mid \mathbf{\Omega}\right) d^{2} \mathbf{x} \approx G_{p, q r}^{v, h}\left(\mathbf{x}_{A}, \mathbf{x}_{B},-t \mid \mathbf{\Omega}\right) .
$$

Equation (16) states that, when no measurements of near sources are available, we can only reconstruct the Green's function $G_{p, q r}^{v, h}\left(\mathbf{x}_{A}, \mathbf{x}_{B},-t \mid \Omega\right)$, which contains the FS multiples. The part of the response that is accurately reconstructed depends on the stationary points that are included in the integration and hence it depends on the size of $\partial \mathbb{D}_{1}$. 


\subsection{Acoustic relations}

For acoustic media the wavefield is characterized by the pressure $p(\mathbf{x}, t)$ and particle velocity $v_{i}(\mathbf{x}, t)$. The pressure and the particle velocity are coupled by the linearized equation of motion, written in the space-frequency domain as,

$$
j \omega \hat{\rho} \hat{v}_{i}+\partial_{i} \hat{p}+2 \hat{\rho} \epsilon_{i j k} \Omega_{j} \hat{v}_{k}=\hat{f}_{i}
$$

and the linearized stress-strain relation

$$
j \omega \hat{\kappa} \hat{p}+\partial_{i} \hat{v}_{i}=\hat{q} .
$$

In (17), again the apparent Coriolis force is included, for a solid rotation with angular velocity $\Omega_{j}$. In (18), $\hat{\kappa}$ is the compressibility which is frequency dependent and complex valued to account for inelastic losses in the medium and $\hat{q}$ is the volume source density of volume injection rate.

Following the same approach as in section 2.2.1, but applying Gauss theorem on the interaction function between two acoustic states $A$ and $B$,

$$
\hat{p}_{A}^{*} \hat{v}_{i, B}+\hat{v}_{i, A}^{*} \hat{p}_{B}
$$

and substituting (17) and (18) in the resulting expression, the acoustic correlation-type reciprocity theorem can be derived. Substituting the acoustic counterparts of states $A$ and $B$, as listed in Table II, into the acoustic correlation-type reciprocity theorem and applying the generalized acoustic source-receiver reciprocity relations, (38) - (41), the acoustic global-scale SI relations can be derived. Alternatively, we can directly rewrite the elastic SI relations, (13) and (16), by replacing the superscripts $h$ by $q, \tau$ by $p$ and leaving out all the subscripts. Note that, comparing equations (17) and (18) with (2) and (3), the sign of the pressure is defined opposite to the that of the stress tensor. Thus, every time we replace $\tau$ by $p$ we also need to introduce a minus sign. Doing so, the acoustic global-scale SI relation reads

$$
\oint_{\partial \mathbb{D}} G^{v, p}\left(\mathbf{x}_{A}, \mathbf{x},-t \mid \boldsymbol{\Omega}\right) * \bar{G}^{p, q}\left(\mathbf{x}_{B}, \mathbf{x}, t \mid \boldsymbol{\Omega}\right) d^{2} \mathbf{x}=G^{v, q}\left(\mathbf{x}_{A}, \mathbf{x}_{B},-t \mid \boldsymbol{\Omega}\right)+\bar{G}^{p, f}\left(\mathbf{x}_{B}, \mathbf{x}_{A}, t \mid \boldsymbol{\Omega}\right)
$$

and the acoustic global-scale SI relation without near offset reads

$$
\int_{\partial \mathbb{D}_{1}} G^{v, p}\left(\mathbf{x}_{A}, \mathbf{x},-t \mid \mathbf{\Omega}\right) * \bar{G}^{p, q}\left(\mathbf{x}_{B}, \mathbf{x}, t \mid \mathbf{\Omega}\right) d^{2} \mathbf{x} \approx G^{v, q}\left(\mathbf{x}_{A}, \mathbf{x}_{B},-t \mid \mathbf{\Omega}\right),
$$

where $v$ and $f$ are in the direction perpendicular to the FS.

In the following we will take $\boldsymbol{\Omega}=\mathbf{0}$. 


\section{GLOBAL-SCALE ACOUSTIC WAVEFIELD MODELING}

In the previous section, we derived relations for obtaining new seismic responses from the correlation of existing global-scale responses. This section deals with the generation of global-scale synthetic earthquake responses (seismograms) with which we can illustrate the derived relations.

We synthesize the seismograms with a simplified 2D acoustic lossless Earth model based on the Preliminary Reference Earth Model (PREM, Dziewonski \& Anderson (1981)). The medium parameters vary only in the radial direction $(r)$. The rotation of the Earth is neglected. Locations on the 2D Earth model are given as function of radius and epicentral distance $(\Delta)$, ranging from -180 to $180^{\circ}$.

Fig. 4(a) shows a graph of the PREM model $P$-wave velocity $c_{P}$ and density $\rho$ as a function of the radius of the Earth. The radius is zero at the center of the Earth and increases to an average thickness of $6371 \mathrm{~km}$ at the Earth's surface. Fig. 4(b) shows our simplified version of the PREM model. As compared to the original PREM model the outermost $3 \mathrm{~km}$ were removed and the discontinuities in the crust and upper mantle were taken away.

We model the wavefield with a staggered finite-difference scheme of the acoustic wave equation (Virieux, 1986). To enable the 2D FD modeling, the PREM model is transformed into a 2D rectangular gridded velocity and density model. We choose the grids small enough ( 2 by $2 \mathrm{~km}$ ) to obviate grid dispersion and ensure stability by satisfying the criteria as derived in Lines et al. (1999).

FD is not the most accurate technique available for synthesizing global-scale seismograms. Still it is the method of choice for our illustration, since consequent inaccuracies are of no harm and the computational affordability and easy adjustability make it a practical scheme to implement.

We generate the responses for state $B$ with the same model as for state $A$, but with the FS removed. Different sources and receivers are placed in the model, to generate all the responses that occur in the acoustic global scale SI relations, (20) and (21). As a source wavelet we use the first derivative of a Gaussian wavelet, with a peak frequency at $0.156 \mathrm{~Hz}$.

A source is initiated at $\Delta=0^{\circ}$ at zero time. Every 0.05 s new values for the wavefield are calculated at every grid point. Starting at $200 \mathrm{~s}$, every $300 \mathrm{~s}$ a snapshot is taken from the 
pressure at every grid point. Fig. 5 shows the evolving pressure distribution throughout the model for state A. The wavefield can be seen to propagate only within the Earth due to the presence of a FS. The wavefield after $200 \mathrm{~s}$ (upper left panel) is not perfectly circular due to the increasing velocity gradient in the crust and mantle. The wavefield is propagating much slower in the transverse direction than in the radial direction. Because of this, part of the wavefield starts to propagate along rays that bend towards the FS. After $500 \mathrm{~s}$ (the upper middle panel) a part of the wavefield has reflected a few times from the FS already. In the same panel, the reflection from the CMB almost hits the FS. Also in this panel, the transmitted wavefield traveling in the radial direction is on its way through the inner core. On the subsequent panels the interaction of the wavefield with the different boundaries results in more and more events. The most pronounced are the FS reflections of the direct wave.

From $\Delta=-180^{\circ}$ to $180^{\circ}$ receivers are placed in the model. In total there are 2880 receivers which corresponds to one receiver per $0.125^{\circ}$ or $14 \mathrm{~km}$. Every $0.4 \mathrm{~s}$ the wavefield is registered at the grid points where the receivers are placed. Fig. 6(a) shows the $v$ registrations of the receivers for state $\mathrm{A}$ and $(\mathrm{b})$ shows the $v$-registrations for state $B$. The first $3000 \mathrm{~s}$ of the registrations are plotted. Only the responses for receivers between $\Delta=0^{\circ}$ and $180^{\circ}$ are shown. The other $180^{\circ}$ of responses are identical because an axi-symmetric model is used. The kinematics of the arrivals can clearly be seen. The amplitude is shown as a color variation from blue (negative) to zero (white) and positive (red). With the current plotting resolution, the true amplitude variation cannot be distinguished. High amplitudes were clipped, such that lower-amplitude arrivals can still be distinguished. The kinematics of the $p$-response of state $A$ (measured just below the FS) and state $B$ look identical with the $v$-response. For this reason the $p$-responses are not shown here.

We compare our FD numerical results with arrival times generated by ray tracing. The computed ray-tracing arrival-times (Knapmeyer, 2004) are plotted, with green crosses, in the same figure (Fig. 6). The events are assigned names according to the conventional (IASPEI) nomenclature (Storchak et al., 2003).

Generally, we see a good correspondence between the FD-modeled response and the ray-tracing arrival times. Since the FD modeling uses an adjusted version of the PREM model (Fig. 4(b)) whereas the ray tracing uses the original PREM model (Fig. 4(a)), the travel-time correspondence is of course not perfect. 
The FD-modeled response for state A (Fig. 6(a)) contains very-high-order FS multiples. The infinite-order FS multiple is a surface wave. This linear event is denoted with 'L'. In the actual Earth the high-order FS multiples cannot be distinguished. This notable difference is because the model we use is $2 \mathrm{D}$ and lossless and without the discontinuities in the upper mantle and crust. Another difference is that refractions, e.g. Pc, are modeled with a faster amplitude decay than they have in the real Earth. This is an artifact of the usage of a rectangular grid. Because of this rectangular grid the interface is not just discontinuous in the radial direction but contains also little variations in the tangential direction by which a wave traveling along the interface is scattered.

In the FD-modeled response for state $B$ (Fig. 6(b)), the reflection due to the change in velocity/density gradient at $570 \mathrm{~km}$ depth (Fig. 4) can be seen. It is labeled with 'a1'. Additionally, low-frequency reflections can be seen, which are labeled with 'a2'. These are artifacts of the absorbing taper at the edges of the model. They were largely filtered out by the application of a high-pass filter.

\section{NUMERICAL ILLUSTRATION}

In section 2, two relations for global-scale SI were derived. Central in both relations is the correlation integral:

$$
\oint_{\partial \mathbb{D}} G^{v, p}\left(\mathbf{x}_{A}, \mathbf{x},-t\right) * \bar{G}^{p, q}\left(\mathbf{x}_{B}, \mathbf{x}, t\right) d^{2} \mathbf{x}
$$

The predicted output differs, depending on whether the entire surface integral is evaluated (20) or the near-offset part (with respect to the receiver position where a source is retrieved) is left out (21). In this section, the correlation integral is numerically tested on the synthesized global responses. The results of the numerical illustration are shown and discussed.

The FD-modeled responses (section 3) can be written as a Green's function convolved with a source wavelet. For compactness we leave out this convolution with the source wavelet. Using the Green's function notation, we modeled responses $G^{v, p}\left(\mathbf{x}, \mathbf{x}_{A}, t\right), \bar{G}^{p, q}\left(\mathbf{x}, \mathbf{x}_{B}, t\right)$, $G^{p, p}\left(\mathbf{x}, \mathbf{x}_{A}, t\right)$ and $\bar{G}^{v, q}\left(\mathbf{x}, \mathbf{x}_{B}, t\right)$. By applying the acoustic source-receiver reciprocity relations, (38) - (41), but without rotation, we have implicitly also modeled the responses $G^{v, p}\left(\mathbf{x}_{A}, \mathbf{x}, t\right), \bar{G}^{p, q}\left(\mathbf{x}_{B}, \mathbf{x}, t\right), G^{v, q}\left(\mathbf{x}_{A}, \mathbf{x}, t\right)$ and $-\bar{G}^{p, f}\left(\mathbf{x}_{B}, \mathbf{x}, t\right)$. Thus we have all the responses needed to illustrate the basic SI relation, (20). 
For testing (21), not the complete FD-modeled responses are used but the Green's functions without the contributions of sources nearby $\mathbf{x}_{A}$ and $\mathbf{x}_{B}$.

The SI relations only contain a correlation of Green's functions. Our synthesized data are Green's functions convolved with a source wavelet, $s(t)$. Including the source wavelets in the basic SI relation in the frequency domain, results in multiplying each term with

$$
\hat{s}^{*}(\omega) \hat{s}(\omega)=|\hat{s}(\omega)|^{2}
$$

which is the power spectrum of the source wavelet. In the time domain each term is convolved with

$$
s(-t) * s(t)
$$

which is the autocorrelation of the source wavelet. Since the FD-modeled result is a Green's function convolved with the source wavelet, we correlate it further with the source wavelet to give it the same phase information as the reconstructed result. Whenever we speak about the FD-modeled Green's function in this section, we mean the FD-modeled Green's function correlated with the source wavelet.

The first step of evaluating (22) numerically is a cross-correlation between the $v$-response measured at $\mathbf{x}_{A}$, with the $p$-response without FS multiples measured at $\mathbf{x}_{B}$. The correlation of responses with $\mathbf{x}_{A}$ at $0^{\circ} \Delta$ and $\mathbf{x}_{B}$ at $-90^{\circ} \Delta$ is depicted on the left-hand side of Fig. 7 . The horizontal axes represent the source positions, ranging from $\Delta=-180^{\circ}$ to $\Delta=180^{\circ}$ The result on the right-hand side of Fig. 7, is a correlation panel. Each trace in the correlation panel corresponds to a correlation of responses due to the same source.

The second step is visualized in Fig. 8. It is an integration over all the source positions, which in the numerical case is a stack of all traces in the correlation panel. As explained by Schuster et al. (2004), Wapenaar et al. (2004) and Snieder (2004), events with stationary phases (events that contain a $\partial_{x} \phi=0$, where $\phi$ is the phase of an event and $\partial_{x}$ denotes the derivative with respect to the source position) in the correlation panel add up during the integration process. All the other amplitudes in the correlation panel cancel by destructive interference. The resulting single trace (middle panel in Fig. 8) has a causal as well as an anti-causal part. According to (20) they should be $G^{v, q}\left(\mathbf{x}_{A}, \mathbf{x}_{B},-t\right)$ and $\bar{G}^{p, f}\left(\mathbf{x}_{B}, \mathbf{x}_{A}, t\right)$, respectively.

Fig.'s 9(a) and 9(b) show the reconstructed time-reversed anticausal Green's function without using near-offset responses for time duration of 3000 and 6000 s, respectively, ac- 
cording to (21). The same amplitude scaling is used for both (a) and (b). Comparing (a) with (b) it can be seen that until $2000 \mathrm{~s}$ the same events are reconstructed and with the same amplitude. At later times, though, we can see in (b) events which cannot be seen in (a). To reconstruct a primary we need at least a first order multiple in the input data (unless there is a delta pulse at time zero in the data). Because the response without FS multiples contains all the significant events before $2000 \mathrm{~s}$ (Fig. 6(b)), an input duration of $3000+2000 \mathrm{~s}$ must be sufficient to reconstruct correctly events occurring before $3000 \mathrm{~s}$.

We have shown that by correlating $G$ with $\bar{G}$ stable amplitudes can be retrieved, independent of the duration of the input responses. In the following, we will use an input duration of $6000 \mathrm{~s}$ to reconstruct all events before $3000 \mathrm{~s}$ with the correct (relative) amplitude.

Continuing our numerical illustration, the described steps of correlation and integration (Fig. 7 and 8) are repeated for all $\mathbf{x}_{B}$ 's around the model. That is, the same response $G^{v, p}\left(\mathbf{x}_{A}, \mathbf{x}, t\right)$ is correlated with $\bar{G}^{p, q}\left(\mathbf{x}_{B}, \mathbf{x}, t\right)$ and subsequently integrated, for varying $\mathbf{x}_{B}$. Doing so we reconstruct responses measured at all epicentral distances, for a source at $\mathbf{x}_{A}$.

Figs. 10(a) and (b) are the first $3000 \mathrm{~s}$ of the time-reversed anticausal reconstructed results for, respectively, the SI relation (20) and the SI relation without near offset (21). The reconstructed results are compared with the expected result, $G^{v, q}\left(\mathbf{x}_{A}, \mathbf{x}_{B}, t\right)$ as shown in Fig. 10(c). This result was obtained by direct modeling of a response at $\mathbf{x}_{A}$ due to sources all around the Earth model.

There is a very good agreement between the different panels. All the FD-modeled events on Fig. 10(c) are reconstructed with SI in both (a) and (b). In (a) and (b) we see, additionally, some noise in the upper left corners and between 50 and $150^{\circ} \Delta$, at around $500 \mathrm{~s}$, an event can be noticed, which is not modeled in (c).

Fig. 11(a) and (b) are the first $3000 \mathrm{~s}$ of the causal reconstructed results for, respectively, the SI relation (20) and the SI relation without near offset (21). Fig. 11(c) shows the expected causal outcome for (20), which is $\bar{G}^{p, f}\left(\mathbf{x}_{B}, \mathbf{x}_{A}, t\right)$. The expected causal outcome for (21) is zero.

At first sight, there is quite a good agreement between the reconstructed responses - Fig. 11(a), and the FD-modeled response (c). Having a closer look at (a) it can be seen that still all events in (c) have been reconstructed. But there are strong artifacts as well. We see especially at earlier times - noisy signal. And before the first 'real' arrival a few events were reconstructed that can not be seen in (c). 
Fig. 11(b) contains the same artifacts as can be seen in (a). But for the artifacts, it is an empty response, as expected.

In appendix B, it is shown that the artifacts in Fig 10 and 11 are due to correlation noise and due to the reconstruction of internal events. It is shown that in our modeling these are present due to numerical errors. When applying SI to actual data, the artifacts could appear due to undersampling of source positions and an unequal illumination of the medium of interest from different angles, respectively.

We draw the conclusion that by applying (20), indeed we have reconstructed $G^{v, q}\left(\mathbf{x}_{A}, \mathbf{x}_{B}, t\right)$ and $\bar{G}^{p, f}\left(\mathbf{x}_{B}, \mathbf{x}_{A}, t\right)$. Also without using the near-offset contribution we were able to make a kinematically good estimation of $G^{v, q}\left(\mathbf{x}_{A}, \mathbf{x}_{B}, t\right)$, but not of $\bar{G}^{p, f}\left(\mathbf{x}_{B}, \mathbf{x}_{A}, t\right)$, as was expected by equation (21). The artifacts can be explained by numerical errors.

We evaluated the relations on a lossless Earth, since in our derivations no losses were assumed. Roux et al. (2005) and Slob et al. (2006) showed that when SI is applied for media with moderate losses, still correct traveltimes but approximate amplitudes are retrieved. Internal events (Appendix B) are amplified as soon as losses are introduced.

\section{TOWARDS APPLICATION}

Thus far, we considered ideal or semi-ideal situations with the availability of sources all around the globe or missing only near-offset sources. In reality, we will not have a dense (earthquake) source distribution in some mid- and far-offsets either. In this section, we show with a stationary phase analysis that also under suboptimal conditions we can still retrieve useful information from the application of the global-scale SI relations.

\subsection{Stationary phase localization}

The working principle of SI can be described as follows. First, observed responses measured at different epicentral distances, and due to the same sources, are correlated. Therewith, higher-order events become lower-order events. At times belonging to the kinematics of the Green's function that would be observed between the two locations between which the responses are measured, stationary phase events appear in the correlation panel. Then by stacking over the correlation panel, the signals at the stationary phases are selected and 
thus the desired Green's function is reconstructed. With a stationary phase event we mean a (part of an) event which contains a stationary phase. In this section, we analyze where these stationary phase events are located in the anticausal correlation panels.

In Fig. 12(a) we show the same anticausal part of the correlation panel as in Fig. 7, which was the result after correlating a response measured at $0^{\circ} \Delta$ with the multiple-free response measured at $-90^{\circ} \Delta$, both due to the same sources at all epicentral distances. To build up the correlation panel, 5760 sources around the model were used to satisfy the Nyquist spatial sampling criterion (appendix B). In Fig. 12(a) the different regions of this correlation panel are indicated with colors. The near-offset region, the region with correlations of responses due to sources which are close to the location $\mathbf{x}_{B}$ where we want to retrieve a source, is indicated with green. The mid- (red) and far-offset (yellow) regions are the correlations of responses due to sources which are at an intermediate to far epicentral distance with respect to the location $\mathbf{x}_{B}$ where we want to retrieve a source.

The locations of the stationary phase events can be recognized in the correlation panel (Fig. 12(a)) by noticing pieces of events where $\partial_{x} \phi=0$. We subsequently stack over the different offset regions to identify the locations of stationary phase events which we might not directly identify visually on the correlation panel. The resulting traces are in Fig. 12(b)(e). With the blue stars on the Earth models above the traces it is indicated from what epicentral distances the responses were used during the stacking.

When we stack over the complete correlation panel we get trace $12(\mathrm{~b})$. When we only stack over the near-offset region, we get trace 12(c). It looks similar to (b). The near offset (in fact the zero offset) contains all the reconstructed events. When we stack over the mid-offset region we catch stationary phase events. The stacking result is trace (d). The far-offset region in the correlation panel contains stationary phase events for constructing events at later times and additional events, as can be seen in the resulting trace (e).

These observations match with the theory as expressed in (20) and (21). Practically, this means that to reconstruct certain events (kinematically correct), we only need to have the part of the (anticausal) correlation panel where the stationary phase arrivals occur. Thus to reconstruct a certain event, we only need responses from sources from a certain range of epicentral distances. In the next section, we clarify the possibilities of applying an SI relation (21) on global-scale seismological data with an example. 


\subsection{PcP retrieval}

Assume we want to retrieve information about the CMB at the question mark location in Fig. 13. We have seismic stations at locations $\mathbf{x}_{A}$ and $\mathbf{x}_{B}$, at $\Delta=0^{\circ}$ and $45^{\circ}$ on either side of the CMB part of interest. But since the Earth is not seismically active near $\mathbf{x}_{A}$ and $\mathbf{x}_{B}$, we cannot directly measure $\mathrm{PcP}$ between $\mathbf{x}_{A}$ and $\mathbf{x}_{B}$. There are 4 obvious possibilities in which we can still retrieve PcP using SI (sources in region 1,2,3 or 4 in Fig. 13). Assume the Earth is seismically active at around $\Delta=-45^{\circ}$ (region 4 ) or at around $90^{\circ}$ (region 1 ), then we can correlate $\mathrm{PcPPcP}$ with $\mathrm{PcP}$ or vice versa to retrieve PcP. If the Earth is seismically active at around $-150^{\circ}$ (region 3 ) or at around $-165^{\circ}$ (region 2), then we can correlate PKP with $\mathrm{PKPPcP}$ or vice versa to retrieve PcP. The epicentral distance values are not exact and only valid for our simplified Earth model (Fig. 4(b)). In our modeling, PKPPcP is a clearer arrival than $\mathrm{PcPPcP}$ (Fig. 6). Thus here we use sources at around $-150^{\circ}$ (region 3 in Fig. 13) to retrieve PcP.

Fig. 14(a) is a small portion of the correlation panel for $\mathbf{x}_{A}$ and $\mathbf{x}_{B}$ at $0^{\circ}$ and $45^{\circ}$ and sources between $-165^{\circ}$ and $-135^{\circ}$. The horizontal axis depicts the locations of the sources. The stationary phase for PcP due to a correlation of PKPPcP (state $A$ ) with PKP (state $B$ ) can be distinguished between $-160^{\circ}$ and $-140^{\circ}$, around $610 \mathrm{~s}$. Fig. 14(b) is just the contribution from the source right at the stationary phase $\left(\Delta=-148^{\circ}\right)$. Since with our modeling we know the exact location of the stationary phase, the contribution from just one source already suffices to retrieve PcP, though we would need to time-window the trace around the estimated $\mathrm{PcP}$ arrival time between $\mathbf{x}_{A}$ and $\mathbf{x}_{B}$, since at other times we have correlation noise. In reality, because of unknown deviations from a 1D reference model, we would need to stack over contributions from sources at an estimated range in which the stationary phase could be located. Fig. 14(c) is a stack of all the source contributions between $-160^{\circ}$ and $-140^{\circ}$, as were plotted in (a). Because of an ideal sampling (1 source per $7 \mathrm{~km}$ ), just PcP is now retrieved and (almost) all correlation noise disappears. Fig. 14(d) is the directly FD-modeled response between $\mathbf{x}_{A}$ and $\mathbf{x}_{B}$. Comparing (c) with (d) we see that the kinematic retrieval of $\mathrm{PcP}$ is exact, but there are (numerical) errors in the phase characteristics.

In reality, we might not have available the responses due to a dense source distributions at around one of the four regions in Fig. 13. We can still retrieve reflection information of 
the question mark location on the CMB in Fig. 13, though, if we have an array of receivers at location $\mathbf{x}_{A}$ and or $\mathbf{x}_{B}$ and combine SI with migration. With an integration over receiver positions, as imbedded in the migration, the stationary phase contribution can be captured, that would otherwise just be missed by having only the response from one source in one of the 4 regions (Artman et al., 2004; Draganov et al., 2004a; Schuster et al., 2004; Wapenaar et al., 2004).

The source sampling around the globe is very good along specific lines, the lithospheric plate boundaries (Fig. 1), but not over large areas. Therefore it is most practical to use receivers that are inline with major earthquake belts and to apply SI in an approximate $2 \mathrm{D}$ sense, like in the above example. For a retrieved elastodynamic response, subsequently, another interferometric step can be used to isolate the surface waves (Halliday et al., 2007).

As input for our SI algorithms we need one response with and one without FS multiples. It is not easy to find a response with all the FS multiples removed, whereas all the other events are undistorted. E.g., a data driven method like surface related multiple removal (SRME) could be used. With the current sampling of sources and receivers on a global scale though, it is not yet feasible. As was shown in this section, SI can still be applied. In the above example the response without FS multiples is easily found by time windowing the response measured at $\mathbf{x}_{B}$ around the time that PKP is expected. A similar approach is taken in Bakulin et al. (2007). Another possibility would be to use wavefield separation to approximate a response without FS multiples (Mehta et al., 2007). Or we could use the response including FS multiples, but due to sources at varying depths. With this approach Draganov et al. (2006) removed ghost events due to reflectors below the sources.

\subsection{Removing undesired events}

Because we know already roughly the velocity and density model of the Earth, we can model in what region of the correlation panels stationary phase events are expected. We can use this knowledge to select these events and to leave out stationary phase events leading to the reconstruction of undesired events. In this subsection, we illustrate this by trying not to reconstruct the very high-order FS multiples.

From an analysis as in Fig. 12 we know that the stationary phase events of the high-order FS multiples occur in the near-offset and a part of the mid-offset region of the correlation 
panels. Fig. 15(a) is a portion of Fig. 10, the time-reversed anticausal reconstruction result. Beyond $\Delta=100^{\circ}$ a dense set of FS multiples obscures the PKPPKP event. We repeat the same application of SI that was used to retrieve the response in Fig. 15(a), but now with leaving out the responses from sources between $\Delta=-24^{\circ}$ and $\left.24^{\circ}\right)$. Fig. $15(\mathrm{~b})$ is a portion of

the result. It indeed does not contain a large part of the higher-order FS multiples, whereas event PKPPKP is preserved. Where PKPPKP was masked by FS multiples in Fig. 15(a) it can clearly be seen in Fig. 15(b). We can achieve this result because stationary phase events leading to events that overlap in the reconstructed result do generally not overlap in the correlation panel. Hence the correlation panel can effectively be used as a domain to filter out (un)desired events.

\section{CONCLUSIONS}

We derived elastodynamic SI relations for transient sources near the closed surface of the Earth. This configuration intrinsically differs from configurations SI has been applied to thus far. The derived relations consist of a correlation integral, in which a response with FS multiples at one receiver is correlated with a response without FS multiples at another receiver. A response without FS multiples is necessary to prevent the occurrence of ghost events and to allow stable amplitude reconstructions in a closed system. Subsequently, the correlation result is integrated over the source locations, thus reconstructing a response between the two receiver locations.

The rotation of the Earth breaks source-receiver reciprocity, but SI relations are still valid. No extra term needs to be evaluated to take the Coriolis force into account.

Using synthesized global-scale earthquake responses we illustrated the acoustic versions of the derived SI relation for a non-rotating Earth. We also tested a slightly different SI relation for the situation when no near-offset responses are available. Both relations gave the expected reconstructed results. When responses due to sources all around the Earth are available both the full Green's function and the Green's function without FS multiples is reconstructed. When no responses are available from sources nearby the receiver position where we want to reconstruct a source only the Green's function including the FS multiples can be reconstructed.

The reconstructions were not without artifacts, viz. noise and additional events. We 
showed that these artifacts could be explained to be due to numerical errors.

For applications to global seismology, only the SI relation without near offset will be of use. Given the distribution of earthquakes around the globe, it will not be possible to reconstruct the complete response between any two receiver positions, since an overall dense source sampling is required. We showed that with a dense source sampling at a specific range of epicentral distances, we can still retrieve specific events between receiver positions. If the location of a stationary phase is known or if an array of receivers is used, one good quality earthquake response could already suffice to retrieve an event between receiver positions.

For the application of SI to actual earthquake data, preprocessing would be necessary. Responses due to different sources would need to be deconvolved for the different source wavelets, normalized for the differences in magnitude and filtered for the differences in source directivity. FS multiples would need to be separated. Also, because in the derivation of SI lossless media are assumed, factors need to be included that account for anelastic losses.

Since the rough velocity and density model of the Earth is already known, we can find out by modeling where in the correlation panel the stationary phases of specific events occur. Subsequently we can determine whether we properly sample certain stationary phase events with a (given) source-receiver configuration. Hence we have a tool to estimate what kind of events in the Green's function between two points on the surface can be reconstructed, given responses from a certain range of epicentral distances with sources. We demonstrated that this knowledge of stationary phases locations in the correlation panel can also be used to filter out undesired events.

\section{Acknowledgments}

This research is supported by The Netherlands Research Centre for Integrated Solid Earth Sciences (ISES) and Netherlands Organisation for Scientific Research (NWO). The authors would like to thank Xander Campman, Evert Slob, Jeannot Trampert and Arie Verdel for insightful comments, Jan Thorbecke for support in implementing the finite difference modeling, Andrey Bakulin and Roel Snieder for a careful review and Roel Snieder we also like to thank for inspiration to extend the theory for rotating media. We used software from the Delphi and CWP consortium. 


\section{References}

Abe, S., Kurashimo, E., Sato, H., Hirata, N., Iwasaki, T., \& Kawanaka, T., 2007. Interferometric seismic imaging of crustal structure using scattered teleseismic waves, Geophysical Research Letters, 34, L19305.

Artman, B., Draganov, D., Wapenaar, K., \& Biondi, B., 2004. Direct migration of passive seismic data, Extended abstracts, P075, EAGE.

Bakulin, A. \& Calvert, R., 2004. Virtual Source: new method for imaging and 4D below complex overburden, Expanded abstracts, 2477-2480, SEG.

Bakulin, A., Mateeva, A., Calvert, R., Jorgensen, P., \& Lopez, J., 2007. Virtual shear source makes shear waves with air guns, Geophysics, $\mathbf{7 2}$.

Berkhout, A.J. \& Verschuur, D.J., 1997. Estimation of multiple scattering by iterative inversion, Part I: Theoretical considerations, Geophysics, 62, 1586-1595.

Campillo, M. \& Paul, A., 2003. Long-range correlations in the diffuse seismic coda, Science, 299, $547-549$.

Claerbout, J.F., 1968. Synthesis of a layered medium from its acoustic transmission response, Geophysics, 33, 264-269.

Dahlen, F.A. \& Tromp, J., 1998. Theoretical Global Seismology, Princeton University Press, Princeton, New Jersey.

de Hoop, A.T., 1988. Time-domain reciprocity theorems for acoustic wave fields in fluids with relaxation, Journal of the Acoustic Society of America, 84, 1877-1882.

Derode, A., Larose, E., Tanter, M., de Rosny, J., Tourin, A., Campillo, M., \& Fink, M., 2003. Recovering the Green's function from field-field correlations in an open scattering medium, Journal of the Acoustic Society of America, 113, 2973-2976.

Draganov, D., Wapenaar, K., Artman, B., \& Biondi, B., 2004. Migration methods for passive seismic data, Expanded abstracts, ST 1.5, SEG.

Draganov, D., Wapenaar, K., \& Thorbecke, J., 2004. Passive seismic imaging in the presence of white noise sources, The Leading Edge, 23, 889-892.

Draganov, D., Wapenaar, K., \& Thorbecke, J., 2006. Seismic interferometry: Reconstructing the earth's reflection response, Geophysics, 71, SI61-SI70.

Draganov, D., Wapenaar, K., Mulder, W., Singer, J., \& Verdel, A., 2007. Retrieval of reflections 
from seismic background-noise measurements, Geophysical Research Letters, 34, L04305.

Dziewonski, M. \& Anderson, Don L., 1981. Preliminary reference earth model, Physics of The Earth and Planetary Interiors, 25, 297-356.

Fan, C., Pavlis, G.L., Weglein, A.B., \& Nita, B.G., 2006. Removing free-surface multiples from teleseismic transmission and constructed reflection responses using reciprocity and the inverse scattering series, Geophysics, 71, SI71-SI78.

Fokkema, J.T. \& van den Berg, P.M., 1993. Seismic Applications of Acoustic Reciprocity, Elsevier.

Gerstoft, P., Sabra, K.G., Roux, P., Kuperman, W.A., \& Fehler, M.C., 2006. Green's functions extraction and surface-wave tomography from microseisms in Southern California, Geophysics, 71.

Halliday, D.F., Curtis, A., Robertsson, J.O.A., \& van Manen, D.J., 2007. Interferometric surfacewave isolation and removal, Geophysics, $\mathbf{7 2}$.

Knapmeyer, M., 2004. TTBox: A Matlab toolbox for the computation of 1D teleseismic travel times, Seismological Research Letters, 75, 726-733.

Kumar, M.R. \& Bostock, M.G., 2006. Transmission to reflection transformation of teleseismic wavefields, Journal of Geophysical Research, 111, B08306.

Levander, A., 2003. USArray design implications for wavefield imaging in the lithosphere and upper mantle, The Leading Edge, 22.

Lines, L. R., Slawinski, R., \& Bording, R.P., 1999. A recipe for stability of finite-difference waveequation computations, Geophysics, 64, 967-969.

Mehta, K., Bakulin, A., Sheiman, J., Calvert, R., \& Snieder, R., 2007. Improving the virtual source method by wavefield seperation, Geophysics, 72, V79-V86.

Pao, Y. H. \& Varatharajulu, V., 1976. Huygens' principle, radiation conditions, and integral formulations for the scattering of elastic waves, Journal of the Acoustic Society of America, 59, 1361-1371.

Roux, P. \& Fink, M., 1997. Experimental evidence in acoustics of the violation of time reversal invariance induced by vorticity, Europhysics Letters, 32, 25-29.

Roux, P., Sabra, K.G., Kuperman, W.A., \& Roux, A., 2005. Ambient noise cross correlation in free space: Theoretical approach, Journal of the Acoustic Society of America, 117, 79-84.

Schuster, G.T., 2001. Theory of daylight/interferometric imaging: tutorial, Extended abstracts, A-32, EAGE. 
Schuster, G.T., Yu, J., Sheng, J., \& Rickett, J., 2004. Interferometric/daylight seismic imaging, Geophysical Journal International, 157, 838-852.

Shapiro, N.M., Campillo, M., Stehly, L., \& Ritzwoller, M.H., 2005. High-resolution surface wave tomography from ambient seismic noise, Science, 307, 1615-1618.

Shiraishi, K., Onishi, K., Ito, S., Aizawa, T., \& Matsuoka, T., 2006. Seismic interferometry with underground moving source, Expanded abstracts, A043, EAGE.

Shragge, J., Artman, B., \& Wilson, C., 2006. Teleseismic shot-profile migration, Geophysics, 71, SI221-SI229.

Slob, E., Draganov, D., \& Wapenaar, K., 2006. GPR without a source, Proceedings of the Eleventh International Conference on Ground Penetrating Radar (Ohio State University, Columbus,OH, 2006).

Snieder, R., 2004. Extracting the Green's function from the correlation of coda waves: a derivation based on stationary phase, Physical Review E, 69, 046610-1-046610-8.

Snieder, R., 2007. Extracting the Green's function of attenuating acoustic media from uncorrelated waves, Journal of the Acoustic Society of America, 121(5), 2637-2643.

Storchak, D. A., Schweitzer, J., \& Bormann, P., 2003. The IASPEI standard seismic phase list, Seismological Research Letters, 74, 761-772.

Verschuur, D.J. \& Berkhout, A.J., 1997. Estimation of multiple scattering by iterative inversion, Part II: Practical aspects and examples, Geophysics, 62, 1596-1611.

Virieux, J., 1986. P-SV wave propagation in heterogeneous media: Velocity-stress finite-difference method, Geophysics, 51, 889-901.

Wapenaar, K., 2006. Nonreciprocal Green's function retrieval by cross correlation, Journal of the Acoustic Society of America, 120, EL7-EL13.

Wapenaar, K. \& Fokkema, J.T., 2006. Green's functions representations for seismic interferometry, Geophysics, 71, SI33-SI46.

Wapenaar, K., Thorbecke, J.W., Draganov, D., \& Fokkema, J.T., 2002. Theory of acoustic daylight imaging revisited, Expanded abstracts, ST 1.5, SEG.

Wapenaar, K., Draganov, D., van der Neut, J., \& Thorbecke, J.W., 2004. Seismic interferometry: a comparison of approaches, Expanded abstracts, ST 1.5, SEG.

Weaver, R.L. \& Lobkis, O.I., 2001. Ultrasonics without a source: thermal fluctuation correlations at MHz frequencies, Physical Review Letters, 87, 134301-1-134301-4. 
Yao, H., van der Hilst, R.D., \& de Hoop, M.V., 2006. Surface-wave array tomography in SE Tibet from ambient seismic noise and two-station analysis: I - phase velocity maps, Geophysical Journal International, 166, 732-744. 


\section{A. DERIVATION OF GENERALIZED SOURCE-RECEIVER RECIPROCITY RE- LATIONS}

In this appendix the generalized source-receiver reciprocity relations for Green's functions are derived. Roux \& Fink (1997) studied the violation of acoustic time-reversal for a rotating medium experimentally. In Dahlen \& Tromp (1998) the general relation between a Green's function in the actual Earth and a Green's function in an Earth with a reversed sense of rotation (the anti-Earth) is given. Here we do the derivations to find the relevant relations for the source and receiver characteristics that are used in this paper.

We start with the Rayleigh-Betti reciprocity theorem of the convolution type:

$$
\begin{aligned}
& \oint_{\partial \mathbb{D}}\left\{\hat{v}_{i, A} \hat{\tau}_{i k, B}-\hat{\tau}_{i k, A} \hat{v}_{i, B}\right\} n_{k} d^{2} \mathbf{x}= \\
& \int_{\mathbb{D}}\left\{-\hat{\tau}_{i j, A} \hat{h}_{i j, B}-\hat{v}_{i, A} \hat{f}_{i, B}+\hat{h}_{i j, A} \hat{\tau}_{i j, B}+\hat{f}_{i, A} \hat{v}_{i, B}\right\} d^{3} \mathbf{x} \\
& \quad-\int_{\mathbb{D}}\left\{2 \rho \epsilon_{i j k} \hat{v}_{i, B} \Omega_{j, B} \hat{v}_{k, A}-2 \rho \epsilon_{i j k} \hat{v}_{i, A} \Omega_{j, A} \hat{v}_{k, B}\right\} d^{3} \mathbf{x},
\end{aligned}
$$

which is found by repeating the derivation for the Rayleigh-Betti reciprocity theorem of the correlation type (section 2.2.1, equation (6)), but by using the interaction function $\left\{\hat{v}_{i, A} \hat{\tau}_{i j, B}-\hat{\tau}_{i j, A} \hat{v}_{i, B}\right\}$ instead of $\left\{-\hat{v}_{i, A}^{*} \hat{\tau}_{i j, B}-\hat{\tau}_{i j, A}^{*} \hat{v}_{i, B}\right\}$, and assuming equal medium parameters in both states.

Using the property $\epsilon_{i j k}=-\epsilon_{k j i}$ we may rewrite the integral with the Coriolis terms, the second integral on the right-hand side of (25), as

$$
-\int_{\mathbb{D}}\left\{2 \rho \epsilon_{i j k} \hat{v}_{i, B} \Omega_{j, B} \hat{v}_{k, A}+2 \rho \epsilon_{i j k} \hat{v}_{i, B} \Omega_{j, A} \hat{v}_{k, A}\right\} d^{3} \mathbf{x}
$$

This integrand will vanish for $\Omega_{j, A}=-\Omega_{j, B}$, i.e., when the medium in state $A$ is rotating opposite to the medium in state $B$. We choose $\Omega_{j, A}=\Omega_{j}$, which is a constant angular velocity, and $\Omega_{j, B}=-\Omega_{j}$. Thus the Rayleigh-Betti reciprocity theorem of the convolution type reduces to

$$
\begin{aligned}
\oint_{\partial \mathbb{D}}\left\{\hat{v}_{i, A} \hat{\tau}_{i k, B}-\hat{\tau}_{i k, A} \hat{v}_{i, B}\right\} n_{k} d^{2} \mathbf{x}= & \\
& \int_{\mathbb{D}}\left\{-\hat{\tau}_{i j, A} \hat{h}_{i j, B}-\hat{v}_{i, A} \hat{f}_{i, B}+\hat{h}_{i j, A} \hat{\tau}_{i j, B}+\hat{f}_{i, A} \hat{v}_{i, B}\right\} d^{3} \mathbf{x} .
\end{aligned}
$$

A domain $\mathbb{D}$ enclosed by a surface $\partial \mathbb{D}$ covers the entire inner space of the Earth, hence $\partial \mathbb{D}$ coincides with the FS (Fig. 16(a)). Only when sources are represented as boundary 
conditions at the FS, the traction vector $\hat{\tau}_{i k} n_{k}$ at $\partial \mathbb{D}$ will not be equal to zero. By making different choices for the sources in state $A$ and $B$ we end up with the desired relations for interchanging source and receiver positions.

One choice is to represent the source in state $A$ as a boundary condition for the traction at the FS. In state $B$ a deformation-rate source is chosen within the domain $\mathbb{D}$. The resulting expressions for the source and wavefield state at $\mathbb{D}$ and $\partial \mathbb{D}$ are given in Table III. Substituting the states of Table III into (27), making use of the sifting property of the delta function and the multiplication property of the Kronecker delta function, yields

$$
\hat{G}_{q r, p}^{\tau, \tau}\left(\mathbf{x}_{B}, \mathbf{x}_{A}, \omega \mid \Omega\right)=\hat{G}_{p, q r}^{v, h}\left(\mathbf{x}_{A}, \mathbf{x}_{B}, \omega \mid-\mathbf{\Omega}\right)
$$

in which $\Omega$ denotes a solid rotation of the medium in $\mathbb{D}$.

Another choice is to represent both the source in state $A$ and the source in state $B$ as boundary condition for the traction at the FS. As there are no sources within $\mathbb{D}$ the first integral on the right-hand side of (27) will vanish. Thus the only term left over from (27) is the surface integral over the wavefield interactions

$$
\oint_{\partial \mathbb{D}}\left\{\hat{v}_{i, A} \hat{\tau}_{i k, B}-\hat{\tau}_{i k, A} \hat{v}_{i, B}\right\} n_{k} d^{2} \mathbf{x}=0 .
$$

The resulting expressions for the wavefield state at $\partial \mathbb{D}$ are given in Table IV. Substituting the states of Table IV into (29), making use of the properties of the delta functions and bringing one of the resulting terms to the other side of the equal sign, we obtain

$$
\hat{G}_{q, p}^{v, \tau}\left(\mathbf{x}_{B}, \mathbf{x}_{A}, \omega \mid \Omega\right)=\hat{G}_{p, q}^{v, \tau}\left(\mathbf{x}_{A}, \mathbf{x}_{B}, \omega \mid-\mathbf{\Omega}\right)
$$

Next, an Earth without FS is assumed. As in Fokkema \& van den Berg (1993) domain $\mathbb{D}$ is chosen to be unbounded. It consists of two parts, as depicted in Fig. 16(b). The inner part is an inhomogeneous medium and contains the sources. The outer part consists of a source-free homogeneous medium and surrounds the first part.

The surface integral in (27) vanishes on account of the radiation conditions (Pao \& Varatharajulu, 1976). Thus, the only term left over from (27) is the volume integral over the source-wavefield interactions

$$
\int_{\mathbb{D}}\left\{-\hat{\tau}_{i j, A} \hat{h}_{i j, B}-\hat{v}_{i, A} \hat{f}_{i, B}+\hat{h}_{i j, A} \hat{\tau}_{i j, B}+\hat{f}_{i, A} \hat{v}_{i, B}\right\} d^{3} \mathbf{x}=0 .
$$

By making various choices for the sources in state $A$ and $B$ we end up with the desired relations for interchanging source and receiver positions. 
One choice is to represent the source in state $A$ as a deformation-rate source, initiating a wavefield at $\mathbf{x}_{A}$. For state $B$ a force source is chosen, initiating a wave at $\mathbf{x}_{B}$. The resulting expressions for the source state and wavefield state are given in Table $\mathrm{V}$.

Substituting the states of Table V into (31) and making use of the properties of the delta functions, yields

$$
\hat{\bar{G}}_{q, p r}^{v, h}\left(\mathbf{x}_{B}, \mathbf{x}_{A}, \omega \mid \boldsymbol{\Omega}\right)=\hat{\bar{G}}_{p r, q}^{\tau, f}\left(\mathbf{x}_{A}, \mathbf{x}_{B}, \omega \mid-\mathbf{\Omega}\right) .
$$

Another choice is to represent both the sources in state $A$ and in state $B$ as deformationrate sources, initiating wavefields at $\mathbf{x}_{A}$ and $\mathbf{x}_{B}$, respectively. The resulting expressions for the source state and wavefield state are given in Table VI.

Substituting the states of Table VI into (31), making use of the properties of the delta functions and omitting the subscript ${ }_{r}$, yields

$$
\hat{\bar{G}}_{q s, p}^{\tau, h}\left(\mathbf{x}_{B}, \mathbf{x}_{A}, \omega \mid \boldsymbol{\Omega}\right)=\hat{\bar{G}}_{p, q s}^{\tau, h}\left(\mathbf{x}_{A}, \mathbf{x}_{B}, \omega \mid-\mathbf{\Omega}\right)
$$

In the time domain, (28), (30), (32) and (33), the generalized source-receiver reciprocity relations, read

$$
\begin{gathered}
G_{q r, p}^{\tau, \tau}\left(\mathbf{x}_{B}, \mathbf{x}_{A}, t \mid \boldsymbol{\Omega}\right)=G_{p, q r}^{v, h}\left(\mathbf{x}_{A}, \mathbf{x}_{B}, t \mid-\mathbf{\Omega}\right), \\
G_{q, p}^{v, \tau}\left(\mathbf{x}_{B}, \mathbf{x}_{A}, t \mid \boldsymbol{\Omega}\right)=G_{p, q}^{v, \tau}\left(\mathbf{x}_{A}, \mathbf{x}_{B}, t \mid-\boldsymbol{\Omega}\right), \\
\bar{G}_{q, p r}^{v, h}\left(\mathbf{x}_{B}, \mathbf{x}_{A}, t \mid \boldsymbol{\Omega}\right)=\bar{G}_{p r, q}^{\tau, f}\left(\mathbf{x}_{A}, \mathbf{x}_{B}, t \mid-\boldsymbol{\Omega}\right) \text { and } \\
\bar{G}_{q s, p}^{\tau, h}\left(\mathbf{x}_{B}, \mathbf{x}_{A}, t \mid \boldsymbol{\Omega}\right)=\bar{G}_{p, q s}^{\tau, h}\left(\mathbf{x}_{A}, \mathbf{x}_{B}, t \mid-\boldsymbol{\Omega}\right) .
\end{gathered}
$$

We can directly find the generalized acoustic source-receiver reciprocity relations by replacing the superscripts $h$ by $q, \tau$ by $-p$ and leaving out all the subscripts. Doing so, the acoustic versions of equations (34) - (37) become

$$
\begin{gathered}
G^{p, p}\left(\mathbf{x}_{B}, \mathbf{x}_{A}, t \mid \Omega\right)=G^{v, q}\left(\mathbf{x}_{A}, \mathbf{x}_{B}, t \mid-\mathbf{\Omega}\right), \\
G^{v, p}\left(\mathbf{x}_{B}, \mathbf{x}_{A}, t \mid \boldsymbol{\Omega}\right)=G^{v, p}\left(\mathbf{x}_{A}, \mathbf{x}_{B}, t \mid-\boldsymbol{\Omega}\right), \\
\bar{G}^{v, q}\left(\mathbf{x}_{B}, \mathbf{x}_{A}, t \mid \boldsymbol{\Omega}\right)=-\bar{G}^{p, f}\left(\mathbf{x}_{A}, \mathbf{x}_{B}, t \mid-\boldsymbol{\Omega}\right) \text { and } \\
\bar{G}^{p, q}\left(\mathbf{x}_{B}, \mathbf{x}_{A}, t \mid \boldsymbol{\Omega}\right)=\bar{G}^{p, q}\left(\mathbf{x}_{A}, \mathbf{x}_{B}, t \mid-\boldsymbol{\Omega}\right) .
\end{gathered}
$$

In a non-rotating medium source-receiver reciprocity holds. The source-receiver reciprocity relations are derived similarly to the derivation of the generalized source-receiver 
reciprocity relations (28), (30), (32) and (33), but with leaving out the argument indicating the sense of rotation. Thus the elastodynamic and acoustic source-receiver reciprocity relations are equations (34) - (37) and (38) - (41), respectively, without the argument indicating the sense of rotation.

\section{B. ARTIFACTS}

\section{B.1. Imperfections}

One large imperfection in our FD-modeled response of state $B$ are the model boundary artifacts. These low-frequency events can be seen in Fig. 10.

With the FD modeling (section 3), both the sources and the receivers were put at approximately $4 \mathrm{~km}$ depth. The resulting response of the model with FS is not optimal. The measured amplitude varies from receiver(set) to receiver(set). There turned out to be a large variation $(2-10 \mathrm{~km})$ in the source/receiver distance to the FS, explaining the differences in amplitude in the measured responses.

The SI algorithm itself is insensitive to mis-positioning of the sources. Normally the mis-positioning gives, e.g., a small delay for the events arriving at $\mathbf{x}_{A}$ and a small delay for the events arriving at $\mathbf{x}_{B}$. After correlation this time-delay is removed. Because of two (numerical) reasons, the mis-positioning of the sources does lead to errors in our numerical implementation, though.

The first reason is that because sources are placed so close to the FS, a mis-positioning has an effect on the effective phase characteristic of the source wavelet. The phase characteristic of the source wavelet arriving at $\mathbf{x}_{B}$ does not change, though, as it is modeled independently in a model without FS.

The second reason is that we used one receiver gather to make receiver gathers at all other epicentral distances. This has as an effect that we correlate traces with each other which are not due to the same source, but are from sources with a different source (mis-)positioning.

\section{B.2. Noise}

According to the theory, by stacking over a correlation panel all amplitudes should interfere destructively, except for the physical events. We found that there was still some noise 
left, most obvious in Fig. 10(a) and (b).

To study the influence of the spatial sampling, we repeat the experiment as visualized in Fig. 7 and 8 for 5 different source samplings. Fig. 17 shows the reconstructed responses using the SI relation without near offset when using (a) 360, (b) 720, (c) 1440, (d) 2880 and (e) 5760 sources around the model. This corresponds to one source every (a) 111, (b) 56, (c) 28 , (d) 14 and (e) $7 \mathrm{~km}$, respectively.

When using 360 sources (a), the 'real' events can hardly be seen through the noise. Using more and more sources, thus increasing the spatial sampling, more and more noise can be seen to disappear. In (e), when using 5760 sources, only a fraction of the noise is left. This number of sources corresponds with the Nyquist spatial sampling criterion for the used frequencies and the minimum velocity in the model.

Here, we have shown that the noise is a function of the source sampling. Thus, this noise is correlation noise, resulting from cross-correlations of all kinds of events that have not shared a common travelpath and thus does not lead to stationary phase events in the correlation panel. With a proper sampling the correlation noise almost completely disappears. The tiny amount left could be due to numerical errors as mentioned in the previous section.

\section{B.3. Additional events}

Here, we analyze the occurrence of additional events. These are reconstructed events which are not in the FD-modeled response. These are sometimes referred to as spurious multiples or non-physical events. They are most obvious in the causal result (Fig. 11(a) and (b)), but can also be seen in the anticausal result (Fig. 10(a) and (b)).

One possibility to reconstruct such additional event would be by a correlation of events as indicated on Fig. 18. We assume an Earth with two velocity contrast (CMB and inner core-outer core boundary), indicated with different levels of grey in the 2D Earth models. A reflection in the state $A$ is correlated with a transmission in the computational state $B$ resulting in an event with the kinematics as if there is a source a the CMB and a direct transmission was measured by a receiver, also at the CMB. On the right-hand side the phase characteristics of the reconstructed event is given, assuming a Gaussian source wavelet. We call such an event that is both initiated and measured at a boundary within the Earth, an internal event. 
To test whether the additional events are actually internal events, we forward-model the response for one source at the $\mathrm{CMB}$ and register the response at receivers placed around the CMB. Also we place a source at the inner core-outer core boundary and measure the response at the same boundary. We plot the responses measured at the CMB upon the responses measured at the inner core-outer core boundary, resulting in Fig. 19(a). This combined response is compared with our previous reconstructed response (Fig. 10 and 11). Fig. 19(b), (c), (d) and (e) depict the first $1000 \mathrm{~s}$ of the causal reconstructed result, the causal result without near offset, the time-reversed anticausal correlation result and the anticausal result without near offset, respectively. It can clearly be seen that the additional events in the causal results indeed have the same kinematics as the events modeled in Fig. 19(a). The additional events in the anticausal result are less visible, but also follow the same kinematics.

From the above test it can be concluded that the additional events are (mainly) internal events that follow from a correlation of events as depicted in Fig. 18.

According to the derived relations for SI, though, no additional events are predicted. So, when the conditions assumed during the derivation of these relations are fulfilled, the additional events should not appear.

With a similar example as in Fig. 18, we can also explain how these additional events could disappear. In Fig. 20 a transmission in the state $A$ is correlated with a reflection in state $B$ resulting in an event with the kinematics as if there is a source a the CMB and a direct transmission was measured by receiver, also at the CMB. On the right-hand side the phase characteristics of the reconstructed event is given, assuming a Gaussian source wavelet. Now comparing Fig. 18 and 20 we see that the reconstructed events have precisely opposite phase characteristics. The summation of both correlation results should cancel these internal events.

Analyzing correlation panels (e.g., as depicted in Fig. 8) it can indeed be seen that these additional events have stationary phase events with opposite phase characteristic. So as the theory predicts and as can be intuitively understood by a simple analysis as above, with a perfect source sampling (perfect illumination from all sides), with no losses within the model and with sufficiently long input responses, these additional events will cancel each other. In our case, it is especially the use of an input duration for which not yet all possible internal multiples have been recorded for state $B$ that leads to unequal amplitudes 
of stationary phase events with opposite phase characteristics. Let us explain. Suppose we call the reconstructed internal event on the right-hand side in Fig. $20 \mathrm{~K}+$. There is only one way to retrieve $\mathrm{K}+$, that is by a correlation of $\mathrm{PKP}$ and $\mathrm{PcP}$, as illustrated in Fig. 20. Suppose we call the reconstructed internal event on the right-hand side in Fig. 18 K-. Fig. 18 is an illustration of only one possibility to retrieve $\mathrm{K}-$, that is by a correlation of PKKP and PKP. But also a correlation of P3KP and PKKP gives $\mathrm{K}$ - and a correlation of P4KP and P3KP gives K-, etc. The amplitude with which we retrieve K- is thus a function of the recording length, or the order of $\mathrm{P}^{*} \mathrm{KP}$ which we include. With our modeling we do not use higher orders than P5KP, while higher orders still have significant amplitude. Thus the sum of $\mathrm{K}+$ and $\mathrm{K}$ - is still a significant deviation from zero.

For real data, the sampling of opposite stationary phase events that should cancel each other, will never be identical. Also, the amplitudes will not be equal because of (different) losses and the duration of the input responses will always be limited. Hence, the (partial) reconstruction of these internal events will be an issue. If we know a-priori where the large contrast exits, we can model where the stationary phases of these internal events will occur in the correlation panels and filter them out.

Besides being a nuisance, these additional events could bring unprecedented possibilities. One of the stationary phase events can be selected and hence a response could be constructed as if induced and measured at, e.g., the CMB. 


\section{Figure captions}

FIG. 1 Distribution of the larger earthquakes over the globe, from 1997 to 2007. Over 15,000 earthquakes with magnitude greater than 5.0 are plotted with clubs, of which the colors depict the magnitude. The map was made with data from the USGS National Earthquake Information Center.

FIG. 2 Configuration for the application of Gauss' integral theorem and for relations derived from Gauss' integral theorem. It depicts the volume of integration, domain $\mathbb{D}$, enclosed by the surface of integration, $\partial \mathbb{D}$, and the outward pointing normal vector $n_{j}$.

FIG. 3 Location of $\mathbf{x}_{A}, \mathbf{x}_{B}$ and $\mathbf{x}$ on a simplified Earth model, with rotation $\Omega_{j}$ opposite to the actual Earth's rotation. A domain $\mathbb{D}$ enclosed by a surface $\partial \mathbb{D}$ covers the entire inner space of the Earth, hence $\partial \mathbb{D}$ coincides with the free surface (denoted as FS). $\hat{\tau}_{i j}$ and $\hat{v}_{i}$ describe the wavefields within $\mathbb{D}$ and at $\partial \mathbb{D}$ due to a source at $\mathbf{x}_{A}$ or $\mathbf{x}_{B}$. (a) State $A$, Earth with FS (free surface); $\mathbf{x}_{A}$ lays approximately at $\partial \mathbb{D}$. (b) State $B$, Earth without FS; $\mathbf{x}_{B}$ lays just within $\mathbb{D}$.

FIG. 4 (a) The compressional-wave velocity $c_{p}$ and density $\rho$ as functions of the radius $r$. Values were taken from the PREM model. With increasing $r$, subsequently the inner core (denoted with A), outer core (B), lower mantle (C), transition zone (D), asthenosphere and lithosphere (E) and atmosphere (F) are encountered. (b) To create this smoothed version of the PREM model the discontinuities in the transition zone, upper mantle and crust were taken out.

FIG. 5 Snapshots of the wavefield spreading through the smoothed PREM model (for state A). The wavefield was initiated by an effective dipole source at $4 \mathrm{~km}$ depth. The snapshots were taken at 200, 500, 800, 1100, 1400, 1700, 2000, 2300 and 2600 seconds, respectively.

FIG. 6 Comparison between FD-modeled responses and ray-tracing arrival-time functions. The horizontal axis shows epicentral distance in degrees and the vertical axis shows time in seconds. The FD-modeled reflection responses of the Earth with (a) and without (b) FS multiples. Ray-tracing arrival times are plotted with green crosses in the same figures. Note that for the FD modeling an adjusted version of the PREM model is used (Fig. 4(b)) whereas for the ray tracing the original PREM model is used (Fig. 4(a)). Note also that positive time axis points downwards, as is conventional in exploration-scale seismology, but 
unconventional in solid Earth seismology.

FIG. 7 Visualization of the integrand of (22). The left panel depicts the time-reversed response of state $A$ recorded at $\Delta=0^{\circ}$, for sources in the range $-180^{\circ}<\Delta<180^{\circ}$. It is convolved with the response of State $B$ recorded at $-90^{\circ}$ epicentral distance (middle panel). The right-hand panel shows the resulting correlation panel.

FIG. 8 Visualization of the integration process. The traces in the correlation panel (left) are summed to produce the trace in the middle panel. On the right, the causal and anticausal part are depicted separately.

FIG. 9 Reconstructed time-reversed anticausal results without using near-offset responses. (a) and (b) are reconstructed traces for $\mathbf{x}_{A}$ at $\Delta=90^{\circ}$ from $\mathbf{x}_{B}$, using an input response duration of respectively $3000 \mathrm{~s}$ and $6000 \mathrm{~s}$.

FIG. 10 Comparison of the time-reversed anticausal reconstructed result (a) and the reconstruction without using near-offset responses (b) with the FD-modeled response (c), which is $G^{v, q}\left(\mathbf{x}_{A}, \mathbf{x}, t\right)$. The horizontal axes show epicentral distance in degrees and the vertical axes shows time in seconds. As the responses are axi-symmetric, only the first $180^{\circ}$ are shown. One degree $\Delta$ corresponds to 8 traces.

FIG. 11 The causal reconstructed result (a), the reconstructed result without using nearoffset responses (b) and the FD-modeled response (c), which is $\bar{G}^{p, f}\left(\mathbf{x}, \mathbf{x}_{A}, t\right)$. The horizontal axes show epicentral distance in degrees and the vertical axes shows time in seconds. For the responses are axi-symmetric, only the first $180^{\circ}$ are shown. One degree $\Delta$ corresponds with 8 traces.

FIG. 12 The time-reversed anticausal correlation panel (a) after correlating a response measured at $\mathbf{x}_{A}$ (at $\Delta=0^{\circ}$ ) with the response without FS multiples measured at $\mathbf{x}_{B}$ $\left(\Delta=-90^{\circ}\right)$. The green, red and yellow colors on the correlation panel denote, respectively, the near-offset, the mid-offset and the far-offset region (with respect to the location $\mathbf{x}_{B}$ where we want to retrieve a source). Stacking over the complete correlation panel, the near-offset, the mid-offset and the far-offset region, gives the traces (b), (c), (d) and (e), respectively. The traces are plotted with the same amplitude scale. The blue stars in the models above the traces indicate source regions from which the responses are observed and used for the reconstruction of the different traces. The green triangles indicate the positions where the responses are measured and between which a response is reconstructed.

FIG. 13 Possible contributions for retrieving PcP between $\mathbf{x}_{A}$ at $\Delta=0^{\circ}$ and $\mathbf{x}_{B}$ at $45^{\circ}$. 
$1,2,3$ and 4 denote seismically active regions at around $\Delta=90,-165^{\circ},-150^{\circ}$ and $-45^{\circ}$ from $\mathbf{x}_{A}$, respectively.

FIG. $14 \mathrm{PcP}$ retrieval between $\mathbf{x}_{A}$ and $\mathbf{x}_{B}$ at $\Delta=45^{\circ}$. (a) portion of time-reversed anticausal correlation panel including the stationary phase for retrieval of PcP. (b) trace at $\Delta=-148^{\circ}$ from the correlation panel. (c) summation of (a) over source positions. (d) FD-modeled response between $\mathbf{x}_{A}$ and $\mathbf{x}_{B}$.

FIG. 15 Comparison of time-reversed anticausal reconstructions. For the upper panel, responses from sources all around the model (but the near offset) were used. For the lower panel, the responses from sources between $\Delta=-24^{\circ}$ and $24^{\circ}$ were left out.

FIG. 16 Two configurations for the application of the elastodynamic reciprocity theorem, one with a closed FS (a), and one unbounded configuration (b).

FIG. 17 The reconstructed result using the SI relation without near offset for $\mathbf{x}_{A}$ at $90^{\circ} \Delta$ from $\mathbf{x}_{B}$ with using respectively (a) 360, (b) 720, (c) 1440, (d) 2880 and (e) 5760 sources around the model.

FIG. 18 Correlation example. The drawings are models of the Earth. The red lines are ray paths of specific events that are measured at a receiver (green triangle) and initiated by a source nearby the Earth's surface (blue star). The correlation of a specific reflection response (drawn in the left-hand model) with a transmission response (in middle model) results in an internal event (right-hand model). The trace on the right-hand side shows the phase-characteristic of the reconstructed event.

FIG. 19 Comparison of the reconstructed additional events with the FD-modeled responses of internal events; (a) the FD-modeled internal events measured and initiated at the CMB as well as measured and initiated at the inner core-outer core boundary; (b) is the causal reconstructed result and (c) is the causal result without near offset (as shown previously in Fig. 11(a) and (b)); (d) is the time-reversed anticausal correlation result and (e) is the result without near offset (as shown previously in Fig. 10(a) and (b)). The first $1000 \mathrm{~s}$ of the responses are shown, because in this time window the additional events were most obvious.

FIG. 20 Correlation example. The drawings are models of the Earth. The red lines are ray paths of specific events that are measured at the FS (green triangle) and initiated by a source nearby the Earth's surface (blue star). The correlation of a transmission response (drawn in left-hand model) with a specific reflection response (in middle model) gives an internal 
event (right-hand model). The traces on the right-hand side show the phase-characteristic of the event reconstructed.

\section{Table captions}

TABLE I Terminology, solid Earth versus exploration-scale seismology

TABLE II States for the elastodynamic reciprocity theorem, in which the source state is a representation for relatively shallow earthquakes.

TABLE III States for the elastodynamic reciprocity theorem with a traction source in state $A$ and a deformation-rate source in state $B$.

TABLE IV States for the elastodynamic reciprocity theorem with traction sources in both states.

TABLE V States for the elastodynamic reciprocity theorem with a deformation-rate source in state $A$ and a force source in state $B$.

TABLE VI States for the elastodynamic reciprocity theorem with deformation-rate sources in both states. 
Figures 


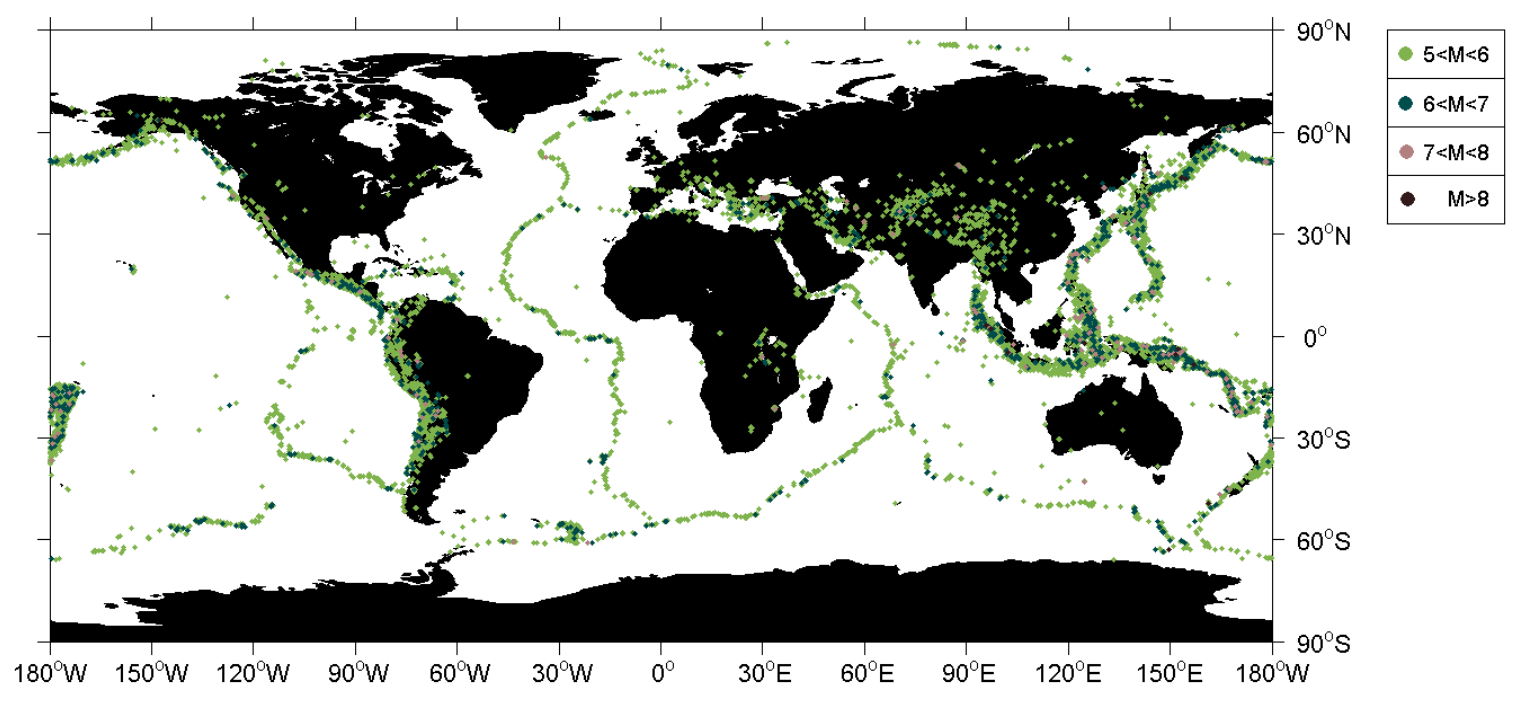

FIG. 1 Distribution of the larger earthquakes over the globe, from 1997 to 2007 . Over 15,000 earthquakes with magnitude greater than 5.0 are plotted with clubs, of which the colors depict the magnitude. The map was made with data from the USGS National Earthquake Information Center. 


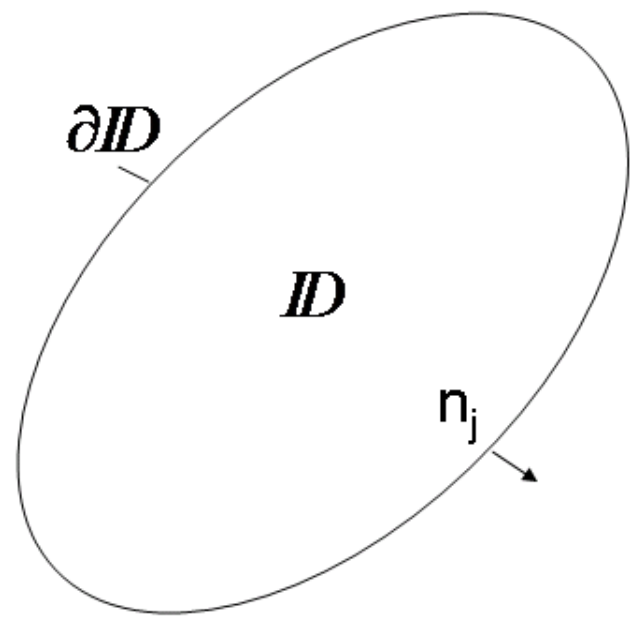

FIG. 2 Configuration for the application of Gauss' integral theorem and for relations derived from Gauss' integral theorem. It depicts the volume of integration, domain $\mathbb{D}$, enclosed by the surface of integration, $\partial \mathbb{D}$, and the outward pointing normal vector $n_{j}$. 


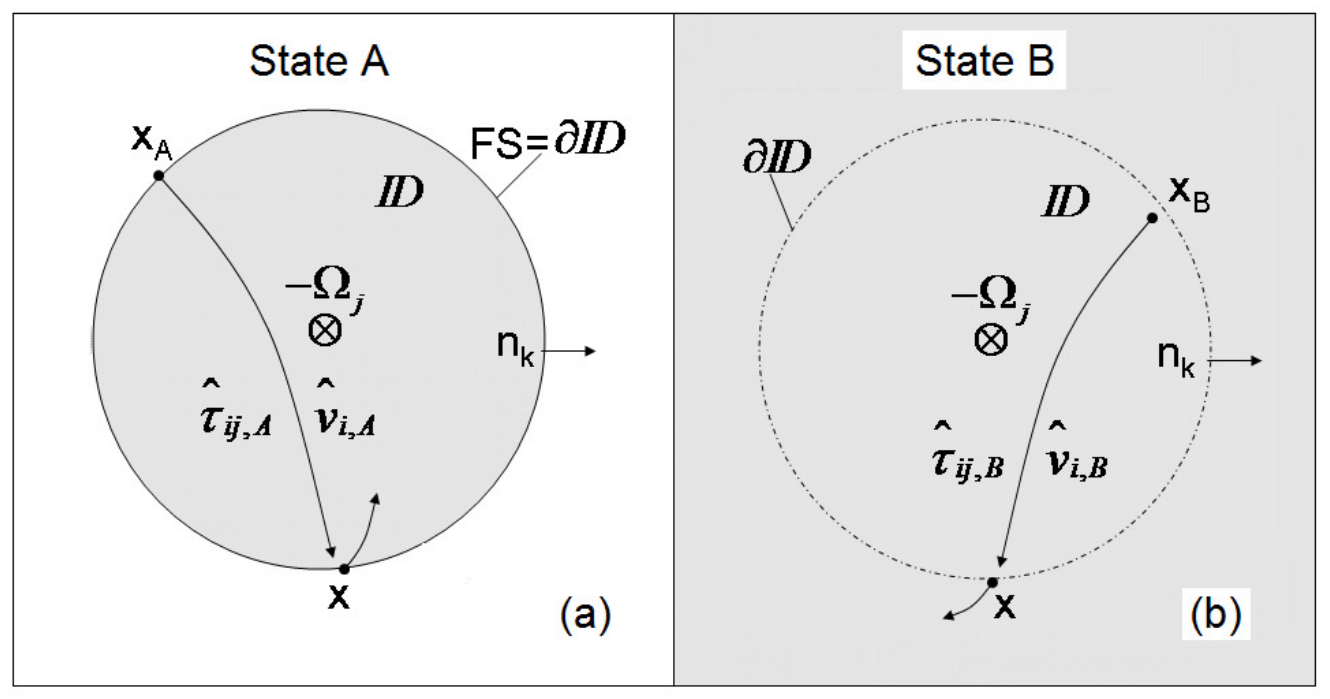

FIG. 3 Location of $\mathbf{x}_{A}, \mathbf{x}_{B}$ and $\mathbf{x}$ on a simplified Earth model, with rotation $\Omega_{j}$ opposite to the actual Earth's rotation. A domain $\mathbb{D}$ enclosed by a surface $\partial \mathbb{D}$ covers the entire inner space of the Earth, hence $\partial \mathbb{D}$ coincides with the free surface (denoted as FS). $\hat{\tau}_{i j}$ and $\hat{v}_{i}$ describe the wavefields within $\mathbb{D}$ and at $\partial \mathbb{D}$ due to a source at $\mathbf{x}_{A}$ or $\mathbf{x}_{B}$. (a) State $A$, Earth with FS (free surface); $\mathbf{x}_{A}$ lays approximately at $\partial \mathbb{D}$. (b) State $B$, Earth without FS; $\mathbf{x}_{B}$ lays just within $\mathbb{D}$. 


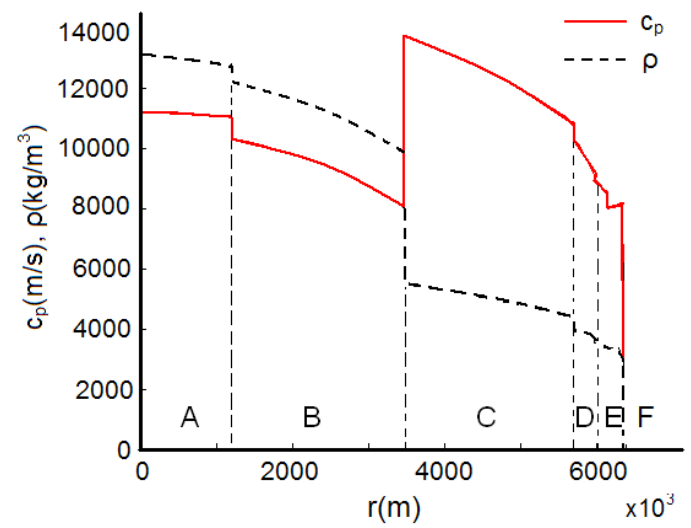

(a)

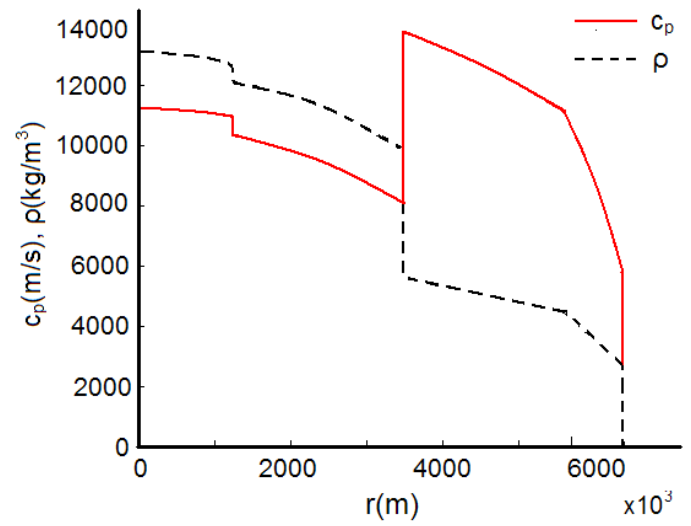

(b)

FIG. 4 (a) The compressional-wave velocity $c_{p}$ and density $\rho$ as functions of the radius $r$. Values were taken from the PREM model. With increasing $r$, subsequently the inner core (denoted with A), outer core (B), lower mantle (C), transition zone (D), asthenosphere and lithosphere (E) and atmosphere $(\mathrm{F})$ are encountered. (b) To create this smoothed version of the PREM model the discontinuities in the transition zone, upper mantle and crust were taken out 


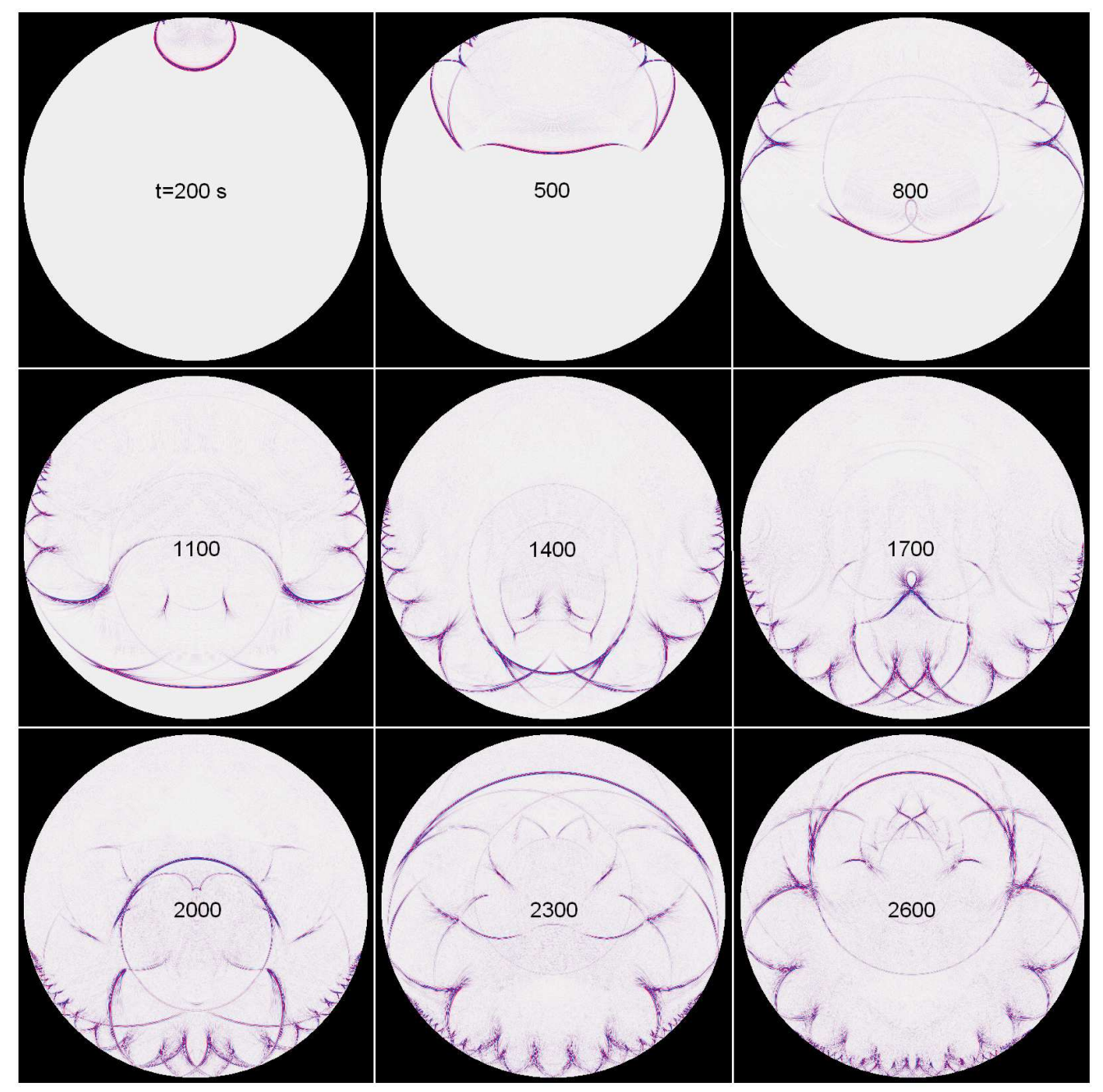

FIG. 5 Snapshots of the wavefield spreading through the smoothed PREM model (for state A). The wavefield was initiated by an effective dipole source at $4 \mathrm{~km}$ depth. The snapshots were taken at 200, 500, 800, 1100, 1400, 1700, 2000, 2300 and 2600 seconds, respectively. 


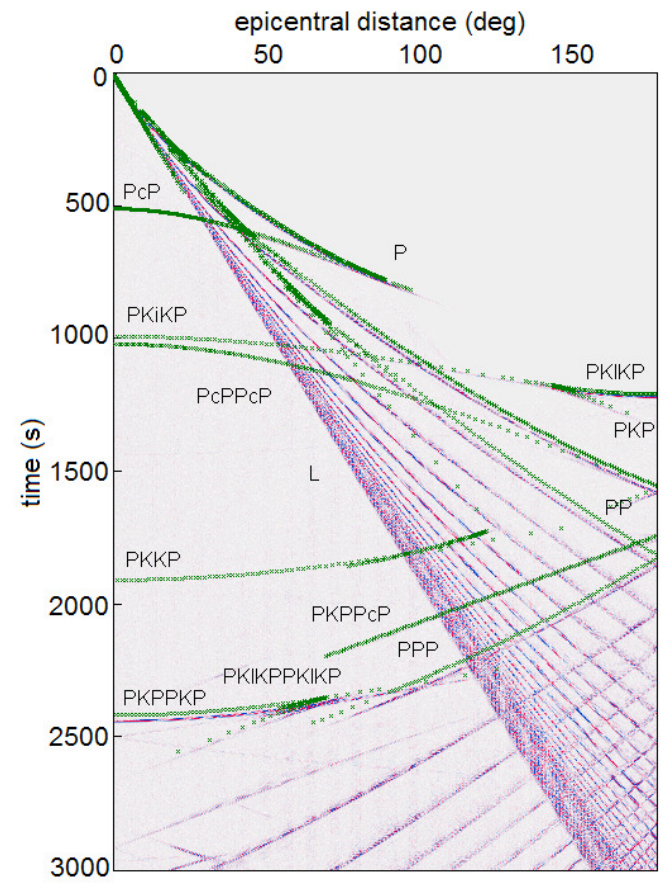

(a)

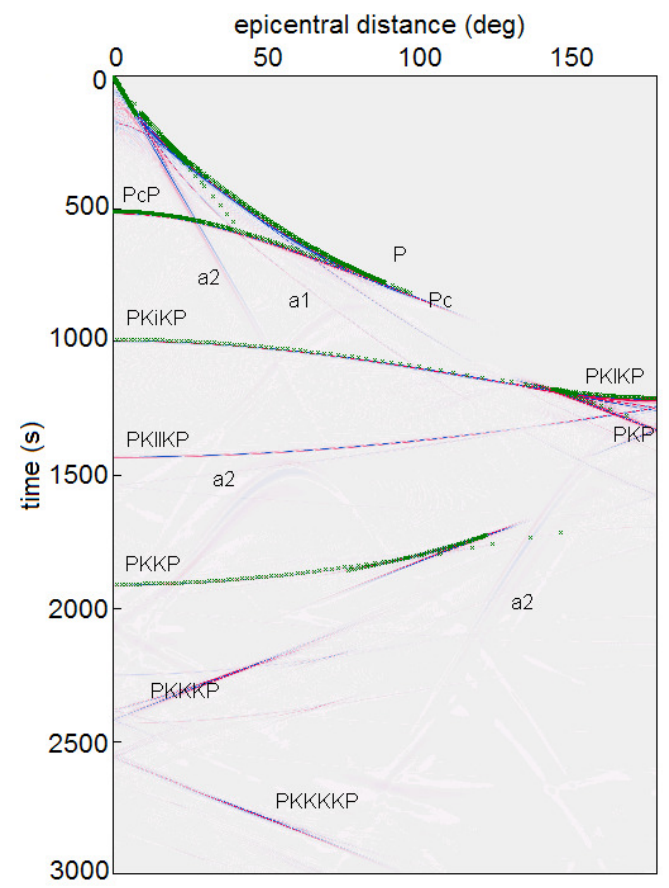

(b)

FIG. 6 Comparison between FD-modeled responses and ray-tracing arrival-time functions. The horizontal axis shows epicentral distance in degrees and the vertical axis shows time in seconds. The FD-modeled reflection responses of the Earth with (a) and without (b) FS multiples. Raytracing arrival times are plotted with green crosses in the same figures. Note that for the FD modeling an adjusted version of the PREM model is used (Fig. 4(b)) whereas for the ray tracing the original PREM model is used (Fig. 4(a)). Note also that positive time axis points downwards, as is conventional in exploration-scale seismology, but unconventional in solid Earth seismology. 


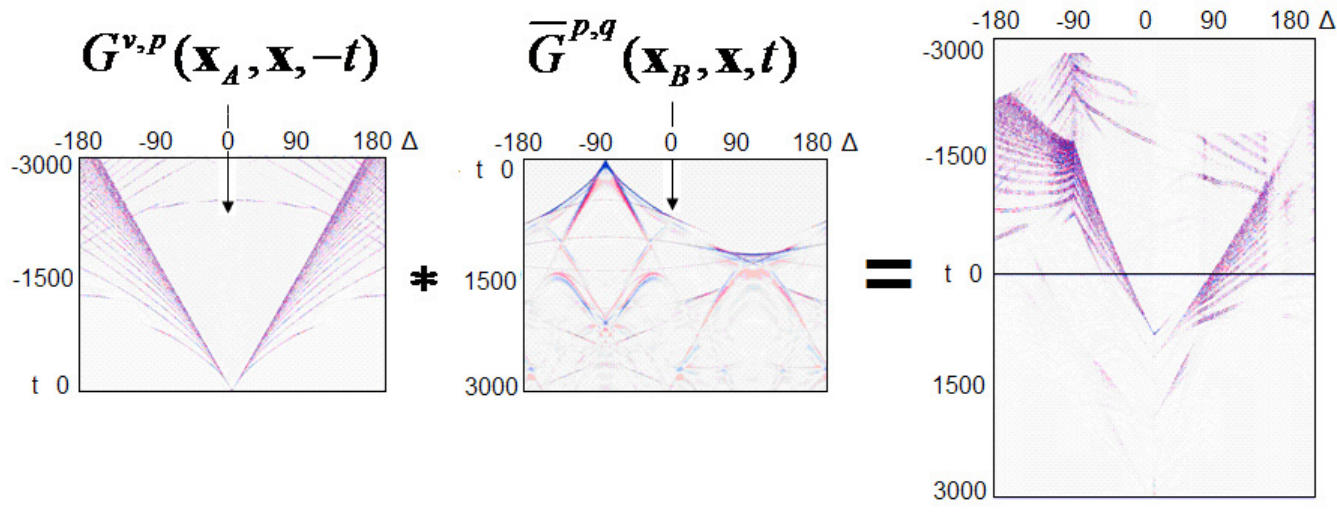

FIG. 7 Visualization of the integrand of (22). The left panel depicts the time-reversed response of state $A$ recorded at $\Delta=0^{\circ}$, for sources in the range $-180^{\circ}<\Delta<180^{\circ}$. It is convolved with the response of State $B$ recorded at $-90^{\circ}$ epicentral distance (middle panel). The right-hand panel shows the resulting correlation panel. 

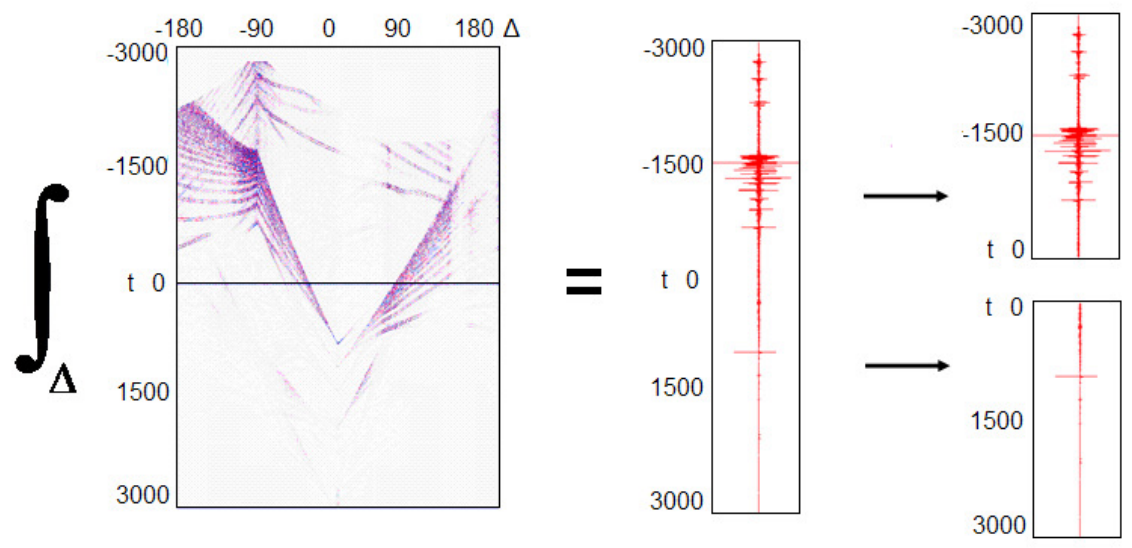

FIG. 8 Visualization of the integration process. The traces in the correlation panel (left) are summed to produce the trace in the middle panel. On the right, the causal and anti-causal part are depicted separately. 


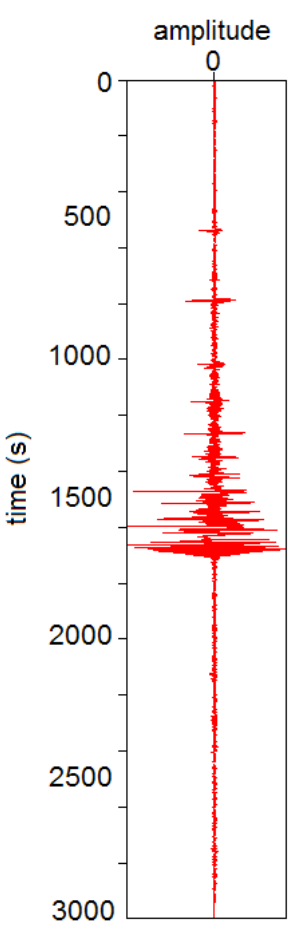

(a)

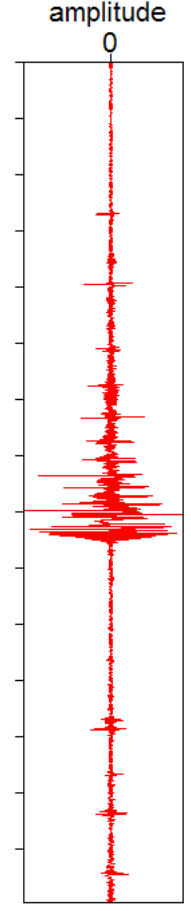

(b)

FIG. 9 Reconstructed time-reversed anticausal results without using near-offset responses. (a) and (b) are reconstructed traces for $\mathbf{x}_{A}$ at $\Delta=90^{\circ}$ from $\mathbf{x}_{B}$, using an input response duration of respectively $3000 \mathrm{~s}$ and $6000 \mathrm{~s}$. 


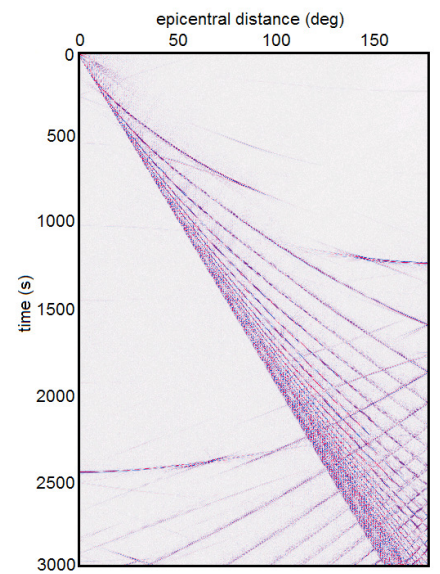

(a)

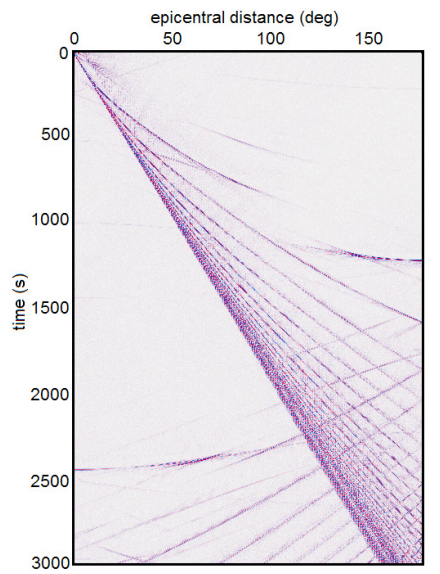

(b)

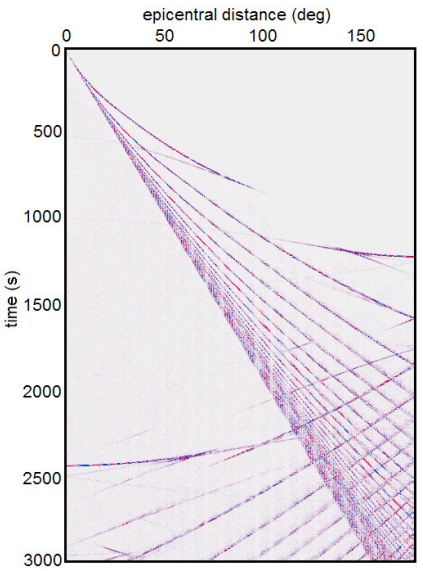

(c)

FIG. 10 Comparison of the time-reversed anticausal reconstructed result (a) and the reconstruction without using near-offset responses (b) with the FD-modeled response (c), which is $G^{v, q}\left(\mathbf{x}_{A}, \mathbf{x}, t\right)$. The horizontal axes show epicentral distance in degrees and the vertical axes shows time in seconds. As the responses are axi-symmetric, only the first $180^{\circ}$ are shown. One degree $\Delta$ corresponds to 8 traces. 


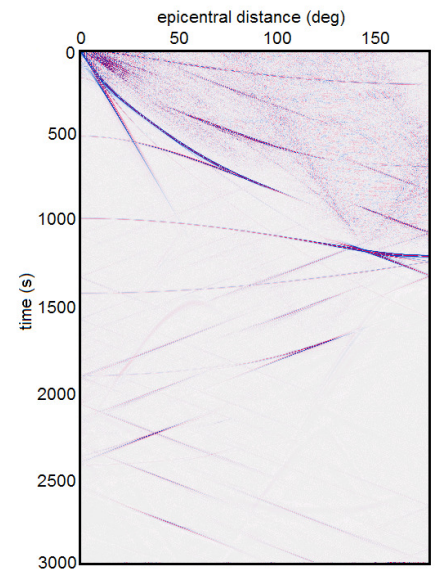

(a)

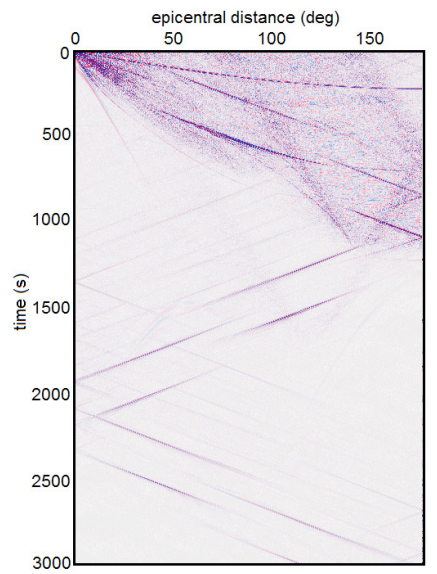

(b)

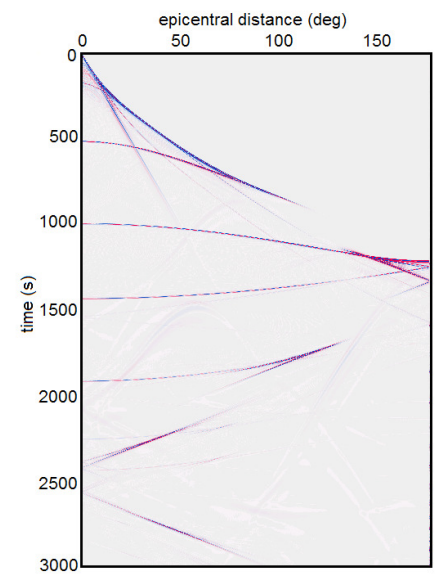

(c)

FIG. 11 The causal reconstructed result (a), the reconstructed result without using near-offset responses (b) and the FD-modeled response (c), which is $\bar{G}^{p, f}\left(\mathbf{x}, \mathbf{x}_{A}, t\right)$. The horizontal axes show epicentral distance in degrees and the vertical axes shows time in seconds. For the responses are axi-symmetric, only the first $180^{\circ}$ are shown. One degree $\Delta$ corresponds with 8 traces. 


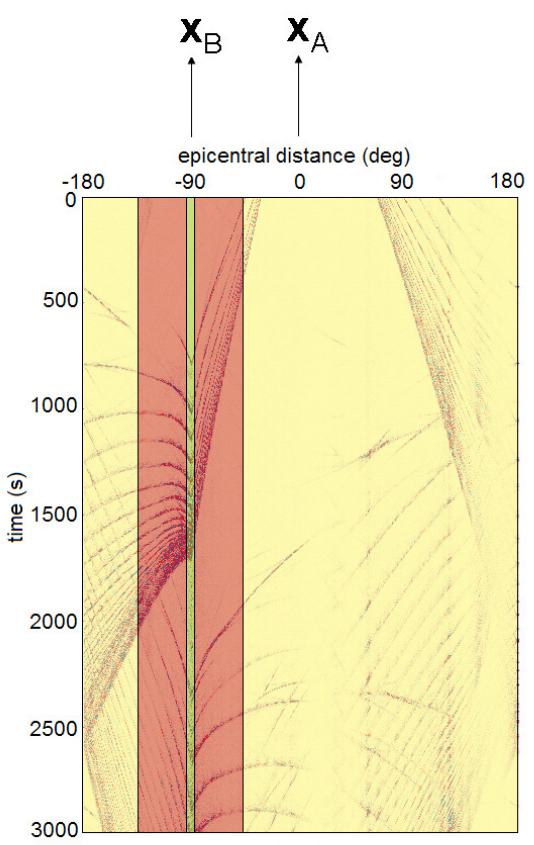

(a)
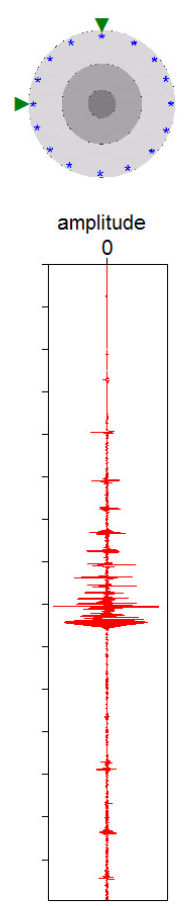

(b)
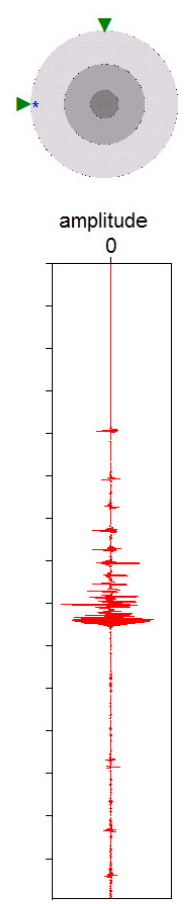

(c)
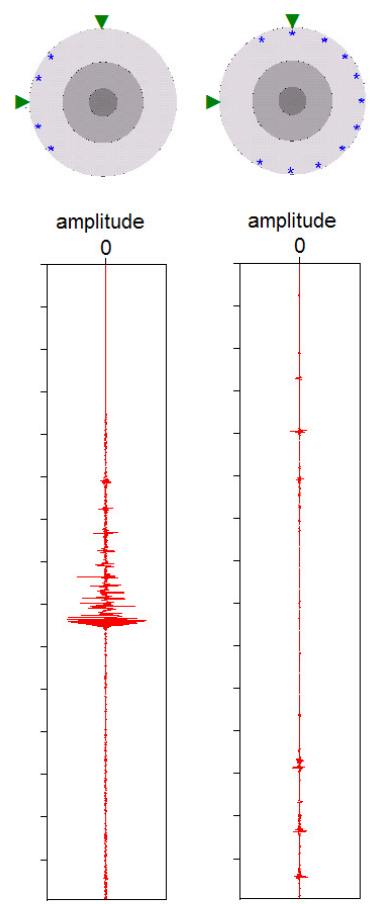

(d)

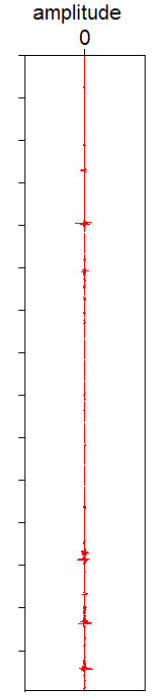

(e)

FIG. 12 The time-reversed anticausal correlation panel (a) after correlating a response measured at $\mathbf{x}_{A}\left(\right.$ at $\left.\Delta=0^{\circ}\right)$ with the response without FS multiples measured at $\mathbf{x}_{B}\left(\Delta=-90^{\circ}\right)$. The green, red and yellow colors on the correlation panel denote, respectively, the near-offset, the mid-offset and the far-offset region (with respect to the location $\mathbf{x}_{B}$ where we want to retrieve a source). Stacking over the complete correlation panel, the near-offset, the mid-offset and the far-offset region, gives the traces (b), (c), (d) and (e), respectively. The traces are plotted with the same amplitude scale. The blue stars in the models above the traces indicate source regions from which the responses are observed and used for the reconstruction of the different traces. The green triangles indicate the positions where the responses are measured and between which a response is reconstructed. 


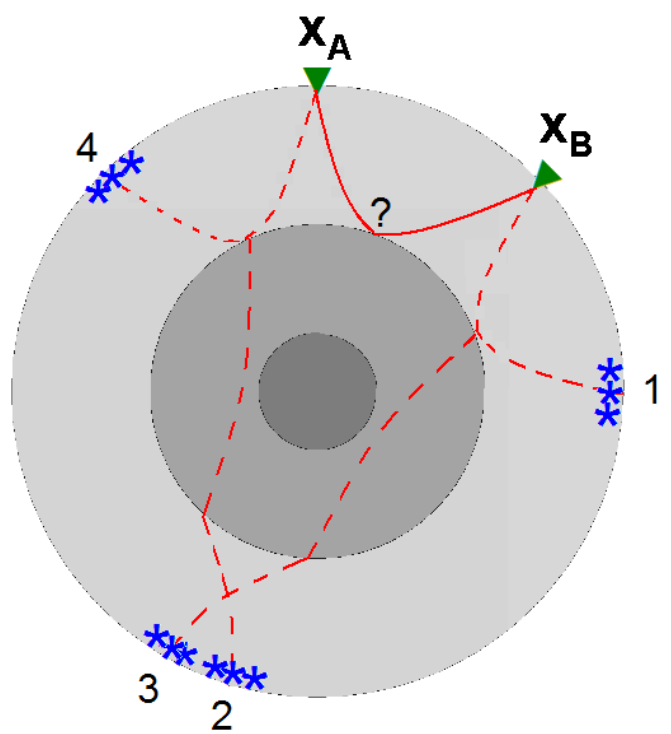

FIG. 13 Possible contributions for retrieving PcP between $\mathbf{x}_{A}$ at $\Delta=0^{\circ}$ and $\mathbf{x}_{B}$ at $45^{\circ} \cdot 1,2,3$ and 4 denote seismically active regions at around $\Delta=90,-165^{\circ},-150^{\circ}$ and $-45^{\circ}$ from $\mathbf{x}_{A}$, respectively. 


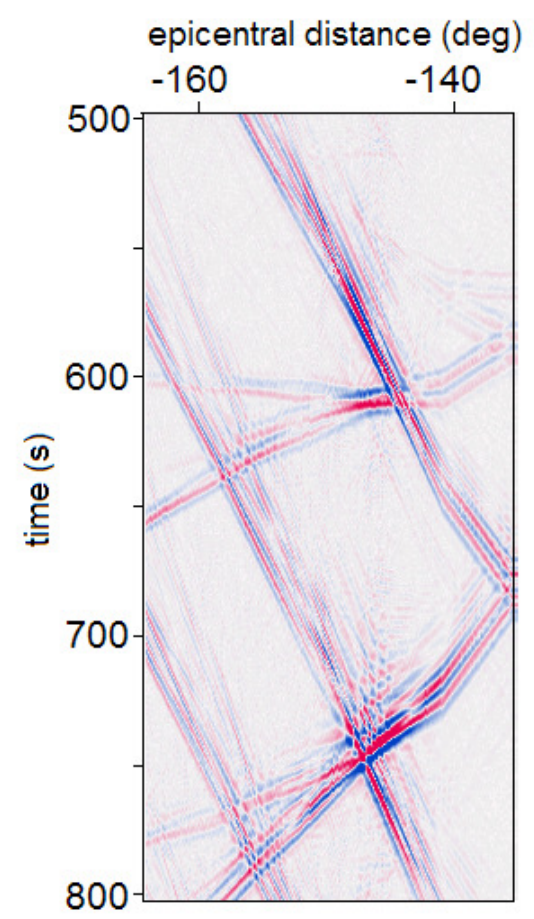

(a)

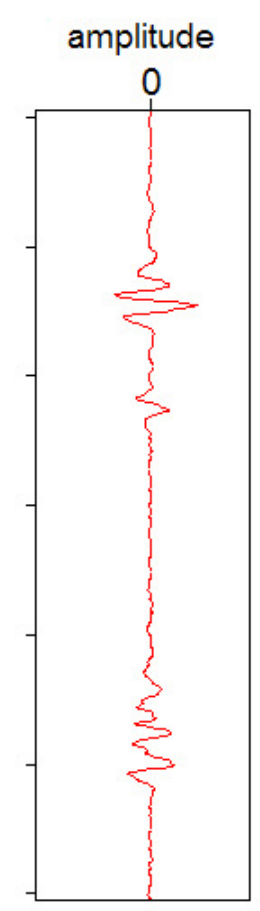

(b)

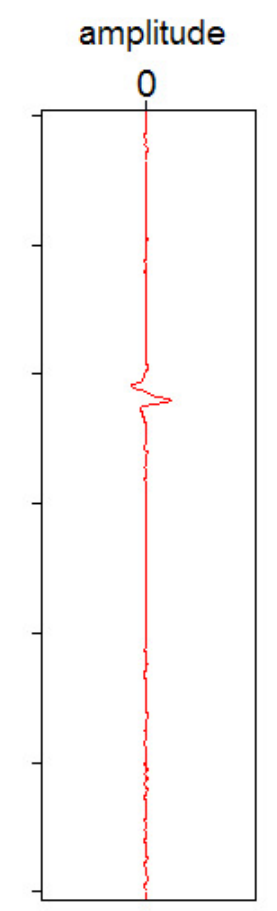

(c)

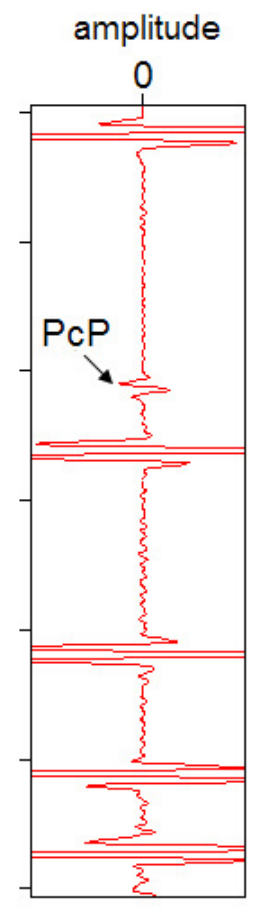

(d)

FIG. 14 PcP retrieval between $\mathbf{x}_{A}$ and $\mathbf{x}_{B}$ at $\Delta=45^{\circ}$. (a) portion of time-reversed anticausal correlation panel including the stationary phase for retrieval of $\mathrm{PcP}$. (b) trace at $\Delta=-148^{\circ}$ from the correlation panel. (c) summation of (a) over source positions. (d) FD-modeled response between $\mathbf{x}_{A}$ and $\mathbf{x}_{B}$. 


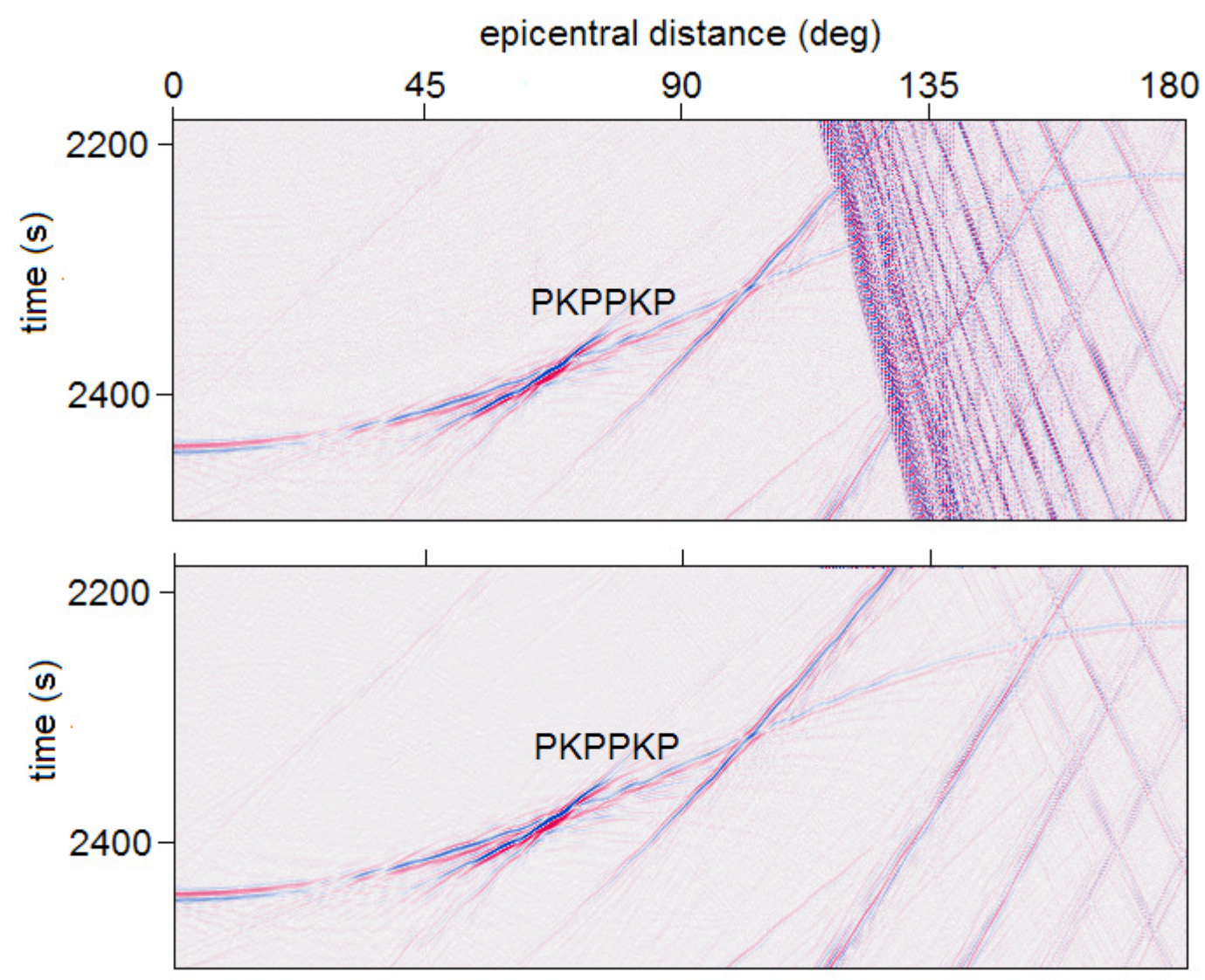

FIG. 15 Comparison of time-reversed anticausal reconstructions. For the upper panel, responses from sources all around the model (but the near offset) were used. For the lower panel, the responses from sources between $\Delta=-24^{\circ}$ and $24^{\circ}$ were left out. 


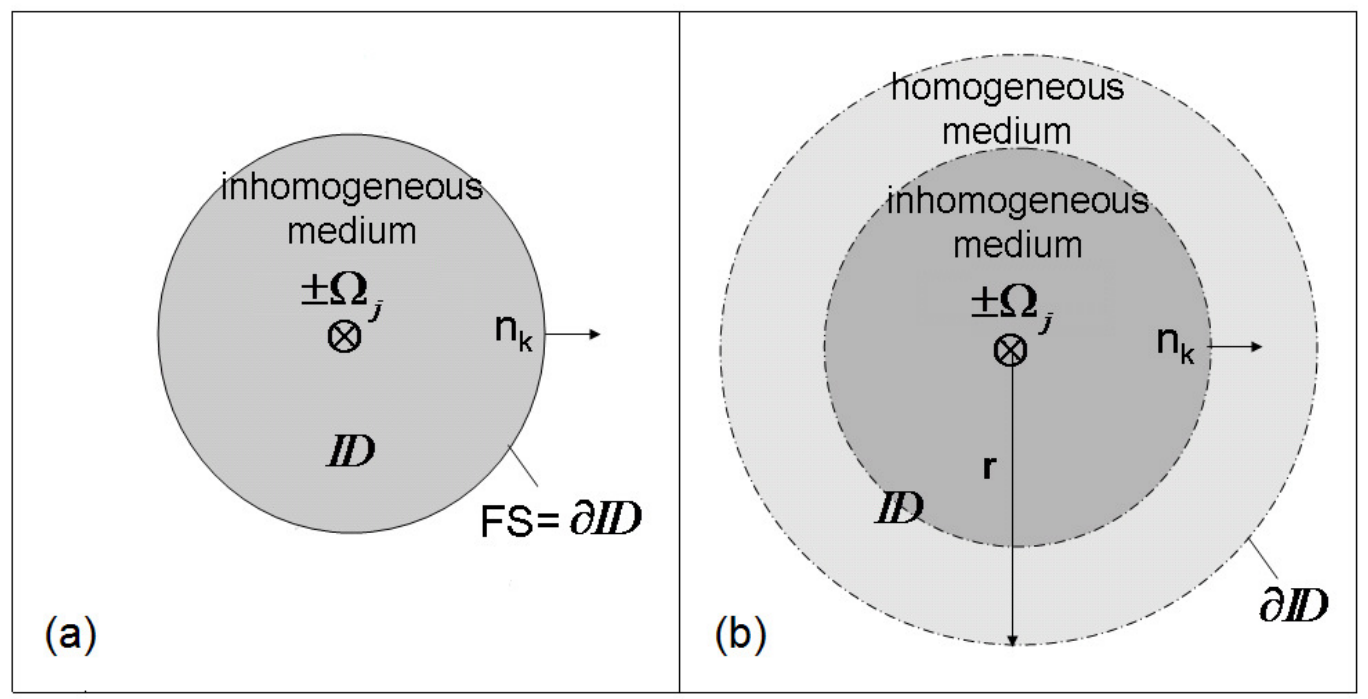

FIG. 16 Two configurations for the application of the elastodynamic reciprocity theorem, one with a closed FS (a), and one unbounded configuration (b). 


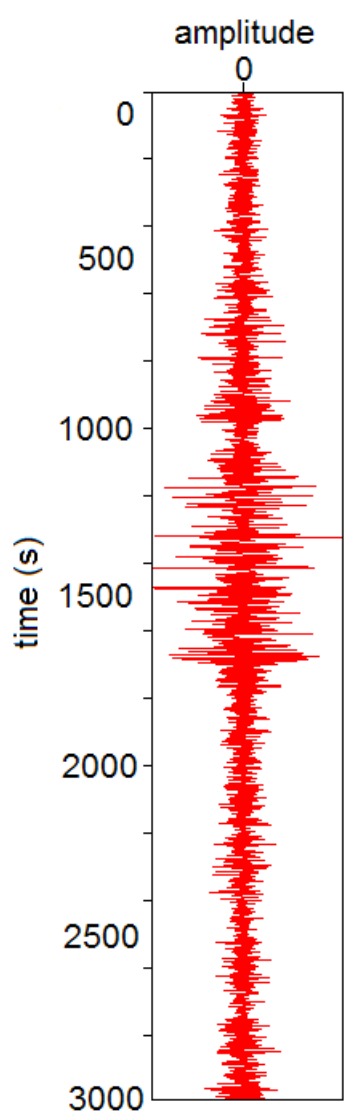

(a)

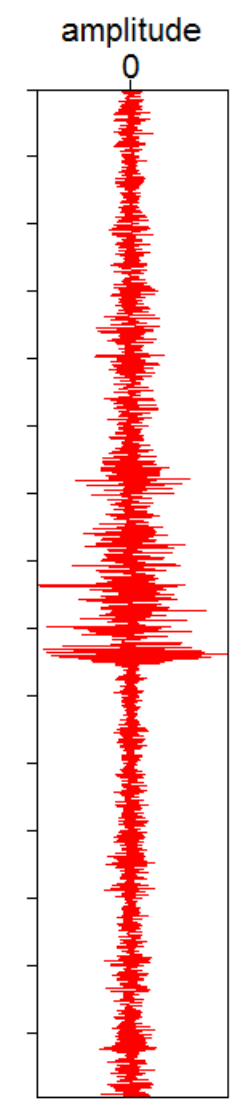

(b)

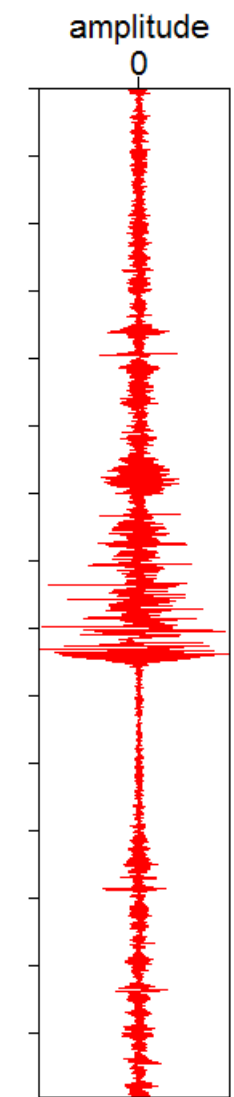

(c)

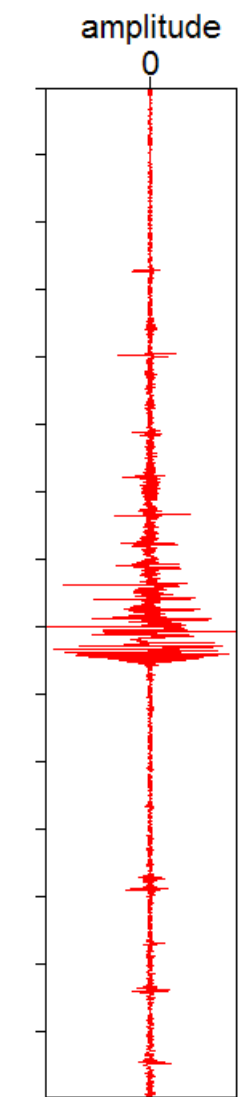

(d)

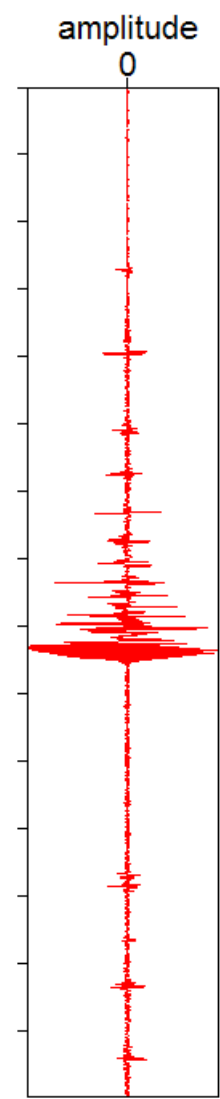

(e)

FIG. 17 The reconstructed result using the SI relation without near offset for $\mathbf{x}_{A}$ at $90^{\circ} \Delta$ from $\mathbf{x}_{B}$ with using respectively (a) 360, (b) 720, (c) 1440, (d) 2880 and (e) 5760 sources around the model. 


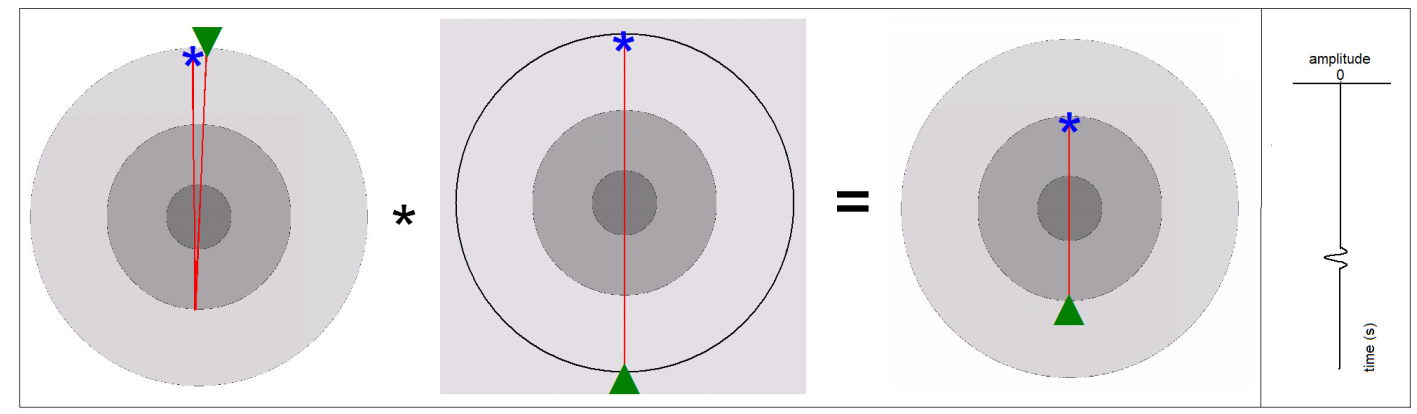

FIG. 18 Correlation example. The drawings are models of the Earth. The red lines are ray paths of specific events that are measured at a receiver (green triangle) and initiated by a source nearby the Earth's surface (blue star). The correlation of a specific reflection response (drawn in the lefthand model) with a transmission response (in middle model) results in an internal event (right-hand model). The trace on the right-hand side shows the phase-characteristic of the reconstructed event. 


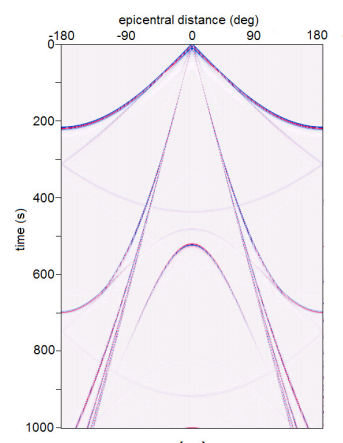

(a)

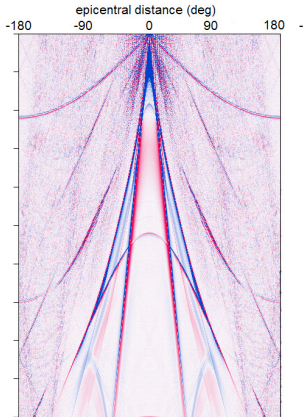

(b)

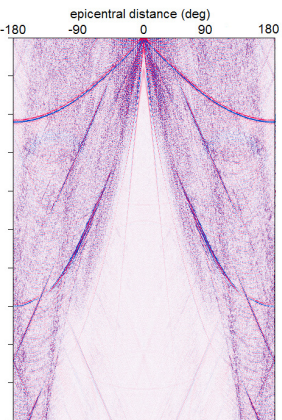

(c)

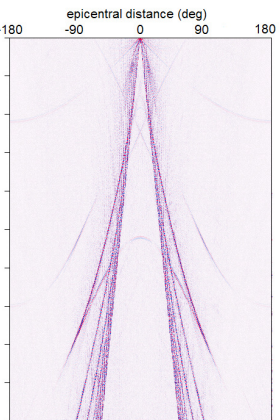

(d)

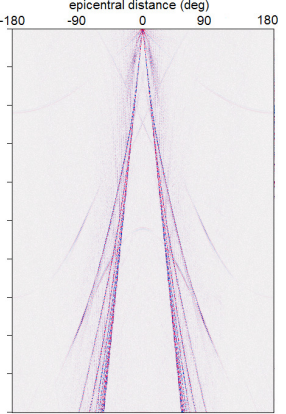

(e)

FIG. 19 Comparison of the reconstructed additional events with the FD-modeled responses of internal events; (a) the FD-modeled internal events measured and initiated at the CMB as well as measured and initiated at the inner core-outer core boundary; (b) is the causal reconstructed result and (c) is the causal result without near offset (as shown previously in Fig. 11(a) and (b)); (d) is the time-reversed anticausal correlation result and (e) is the result without near offset (as shown previously in Fig. 10(a) and (b)). The first $1000 \mathrm{~s}$ of the responses are shown, because in this time window the additional events were most obvious. 


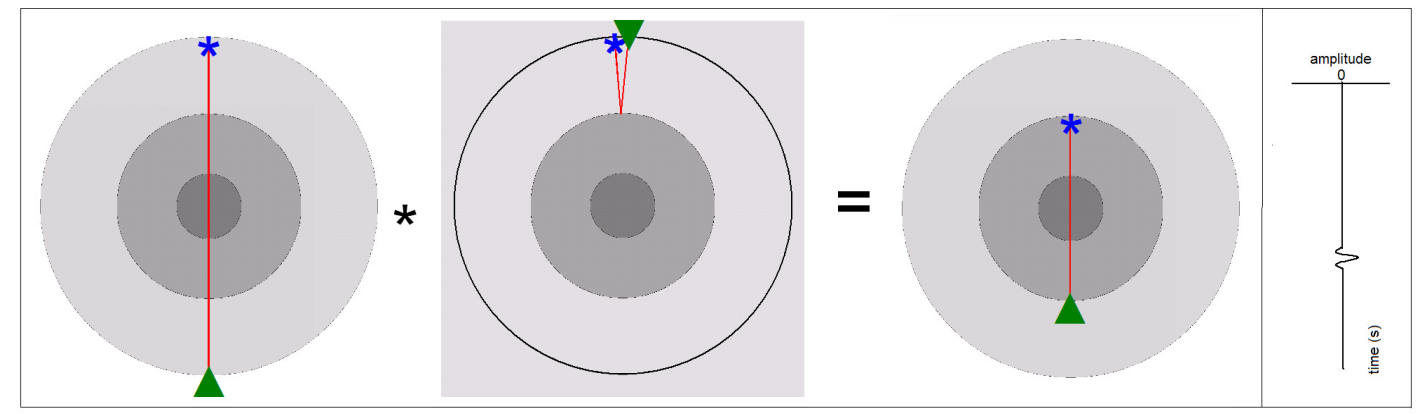

FIG. 20 Correlation example. The drawings are models of the Earth. The red lines are ray paths of specific events that are measured at the FS (green triangle) and initiated by a source nearby the Earth's surface (blue star). The correlation of a transmission response (drawn in left-hand model) with a specific reflection response (in middle model) gives an internal event (right-hand model). The traces on the right-hand side show the phase-characteristic of the event reconstructed. 
Tables 


\begin{tabular}{|l|l|}
\hline solid Earth & exploration \\
\hline source time function & source wavelet \\
epicentral distance & offset \\
seismogram & trace \\
station & receiver \\
seismic event & source \\
phase & event \\
\hline
\end{tabular}

TABLE I Terminology, solid Earth versus exploration-scale seismology 


\begin{tabular}{|c|c|c|c|}
\hline & & $\begin{array}{l}\text { State } A \text { : } \\
\text { free surface }\end{array}$ & $\begin{array}{l}\text { State } B \text { : } \\
\text { no free surface }\end{array}$ \\
\hline $\begin{array}{l}\text { wavefield } \\
\mathbf{x} \in \mathbb{D}\end{array}$ & $\hat{\tau}_{i j}$ & $\left\{\begin{array}{l}\hat{G}_{i j, p}^{\tau, \tau}\left(\mathbf{x}, \mathbf{x}_{A}, \omega \mid-\boldsymbol{\Omega}\right) \\
\hat{G}_{i, p}^{v, \tau}\left(\mathbf{x}, \mathbf{x}_{A}, \omega \mid-\boldsymbol{\Omega}\right)\end{array}\right.$ & $\begin{array}{l}\hat{\bar{G}}_{i j, q r}^{\tau, h}\left(\mathbf{x}, \mathbf{x}_{B}, \omega \mid-\boldsymbol{\Omega}\right) \\
\hat{\bar{G}}_{i, q r}^{v, h}\left(\mathbf{x}, \mathbf{x}_{B}, \omega \mid-\boldsymbol{\Omega}\right)\end{array}$ \\
\hline $\begin{array}{c}\text { wavefield } \\
\mathbf{x} \in \partial \mathbb{D}\end{array}$ & $\begin{array}{l}\hat{\tau}_{i k} n_{k} \\
\hat{v}_{i}\end{array}$ & $\begin{array}{l}\delta\left(\mathbf{x}-\mathbf{x}_{A}\right) \delta_{i p} \\
\hat{G}_{i, p}^{v, \tau}\left(\mathbf{x}, \mathbf{x}_{A}, \omega \mid-\mathbf{\Omega}\right)\end{array}$ & $\begin{array}{l}\hat{\bar{G}}_{i, q r}^{\tau, h}\left(\mathbf{x}, \mathbf{x}_{B}, \omega \mid-\boldsymbol{\Omega}\right) \\
\hat{\bar{G}}_{i, q r}^{v, h}\left(\mathbf{x}, \mathbf{x}_{B}, \omega \mid-\boldsymbol{\Omega}\right)\end{array}$ \\
\hline $\begin{array}{l}\text { source } \\
\qquad \mathbf{x} \in \mathbb{D}\end{array}$ & $\begin{array}{l}\hat{h}_{i j} \\
\hat{f}_{i}\end{array}$ & 0 & $\begin{array}{l}\delta\left(\mathbf{x}-\mathbf{x}_{B}\right) \delta_{i q} \delta_{j r} \\
0\end{array}$ \\
\hline
\end{tabular}

TABLE II States for the elastodynamic reciprocity theorem, in which the source state is a representation for relatively shallow earthquakes. 


\begin{tabular}{|l|l|l|l|}
\cline { 2 - 4 } \multicolumn{1}{l|}{} & State $A:$ & State $B:$ \\
free surface & free surface \\
\hline wavefield & $\hat{\tau}_{i j}$ & $\hat{G}_{i j, p}^{\tau, \tau}\left(\mathbf{x}, \mathbf{x}_{A}, \omega \mid \boldsymbol{\Omega}\right)$ & $\hat{G}_{i j, q r}^{\tau, h}\left(\mathbf{x}, \mathbf{x}_{B}, \omega \mid-\boldsymbol{\Omega}\right)$ \\
$\mathbf{x} \in \mathbb{D}$ & $\hat{v}_{i}$ & $\hat{G}_{i, p}^{v, \tau}\left(\mathbf{x}, \mathbf{x}_{A}, \omega \mid \boldsymbol{\Omega}\right)$ & $\hat{G}_{i, q r}^{v, h}\left(\mathbf{x}, \mathbf{x}_{B}, \omega \mid-\boldsymbol{\Omega}\right)$ \\
\hline wavefield & $\hat{\tau}_{i k} n_{k}$ & $\delta\left(\mathbf{x}-\mathbf{x}_{A}\right) \delta_{i p}$ & 0 \\
$\mathbf{x} \in \partial \mathbb{D}$ & $\hat{v}_{i}$ & $\hat{G}_{i, p}^{v, \tau}\left(\mathbf{x}, \mathbf{x}_{A}, \omega \mid \boldsymbol{\Omega}\right)$ & $\hat{G}_{i, q r}^{v, h}\left(\mathbf{x}, \mathbf{x}_{B}, \omega \mid-\boldsymbol{\Omega}\right)$ \\
\hline source & $\hat{h}_{i j}$ & 0 & $\delta\left(\mathbf{x}-\mathbf{x}_{B}\right) \delta_{i q} \delta_{j r}$ \\
$\mathbf{x} \in \mathbb{D}$ & $\hat{f}_{i}$ & 0 & 0 \\
\hline
\end{tabular}

TABLE III States for the elastodynamic reciprocity theorem with a traction source in state $A$ and a deformation-rate source in state $B$. 


\begin{tabular}{|c|c|c|c|}
\hline & & $\begin{array}{l}\text { State } A \text { : } \\
\text { free surface }\end{array}$ & $\begin{array}{l}\text { State } B \text { : } \\
\text { free surface }\end{array}$ \\
\hline wavefield & $\hat{\tau}_{i k} n_{k}$ & $\delta\left(\mathbf{x}-\mathbf{x}_{A}\right) \delta_{i p}$ & $\delta\left(\mathbf{x}-\mathbf{x}_{B}\right) \delta_{i q}$ \\
\hline $\mathbf{x} \partial \mathbb{D}$ & $\hat{v}_{i}$ & $\hat{G}_{i, p}^{v, \tau}\left(\mathbf{x}, \mathbf{x}_{A}, \omega \mid \mathbf{\Omega}\right)$ & $\hat{G}_{i, q}^{v, \tau}\left(\mathbf{x}, \mathbf{x}_{B}, \omega \mid-\boldsymbol{\Omega}\right)$ \\
\hline
\end{tabular}

TABLE IV States for the elastodynamic reciprocity theorem with traction sources in both states. 


\begin{tabular}{|l|l|l|l|}
\cline { 3 - 4 } \multicolumn{1}{c|}{} & \multicolumn{1}{l|}{$\begin{array}{l}\text { State } A: \\
\text { no free surface }\end{array}$} & State $B:$ \\
no free surface
\end{tabular}

TABLE V States for the elastodynamic reciprocity theorem with a deformation-rate source in state $A$ and a force source in state $B$. 


\begin{tabular}{|l|l|l|l|}
\cline { 2 - 4 } \multicolumn{1}{c|}{} & \multicolumn{1}{l|}{$\begin{array}{l}\text { State } A: \\
\text { no free surface }\end{array}$} & State $B:$ \\
no free surface \\
\hline wavefield & $\hat{\tau}_{i j}$ & $\hat{\bar{G}}_{i j, p r}^{\tau, h}\left(\mathbf{x}, \mathbf{x}_{A}, \omega \mid \boldsymbol{\Omega}\right)$ & $\hat{\bar{G}}_{i j, q s}^{\tau, h}\left(\mathbf{x}, \mathbf{x}_{B}, \omega \mid-\boldsymbol{\Omega}\right)$ \\
$\mathbf{x} \in \mathbb{D}$ & $\hat{v}_{i}$ & $\hat{\bar{G}}_{i, p r}^{v, h}\left(\mathbf{x}, \mathbf{x}_{A}, \omega \mid \boldsymbol{\Omega}\right)$ & $\hat{\bar{G}}_{i, q s}^{v, h}\left(\mathbf{x}, \mathbf{x}_{B}, \omega \mid-\boldsymbol{\Omega}\right)$ \\
\hline source & $\hat{h}_{i j}$ & $\delta\left(\mathbf{x}-\mathbf{x}_{A}\right) \delta_{i p} \delta_{j r}$ & $\delta\left(\mathbf{x}-\mathbf{x}_{B}\right) \delta_{i q} \delta_{j s}$ \\
$\mathbf{x} \in \mathbb{D}$ & $\hat{f}_{i}$ & 0 & 0 \\
\hline
\end{tabular}

TABLE VI States for the elastodynamic reciprocity theorem with deformation-rate sources in both states. 